Subspace electrode selection methodology for the reduction of the effect of uncertain conductivity values in the EEG dipole localization: a simulation study using a patient-specific head model

This article has been downloaded from IOPscience. Please scroll down to see the full text article.

2012 Phys. Med. Biol. 571963

(http://iopscience.iop.org/0031-9155/57/7/1963)

View the table of contents for this issue, or go to the journal homepage for more

Download details:

IP Address: 157.193.59.150

The article was downloaded on 29/05/2012 at 15:07

Please note that terms and conditions apply. 


\title{
Subspace electrode selection methodology for the reduction of the effect of uncertain conductivity values in the EEG dipole localization: a simulation study using a patient-specific head model
}

\author{
G Crevecoeur $^{1}$, V Montes Restrepo ${ }^{2}$ and S Staelens ${ }^{2,3}$ \\ ${ }^{1}$ Department of Electrical Energy, Systems and Automation, Ghent University, \\ Sint-Pietersnieuwstraat 41, B-9000 Ghent, Belgium \\ 2 Medical Image and Signal Processing Research Group, Faculty of Engineering and \\ Architecture, Ghent University-IBBT, Ghent, Belgium \\ ${ }^{3}$ Molecular Imaging Center Antwerp, Faculty of Medicine, Antwerp University, Antwerp, \\ Belgium \\ E-mail: Guillaume.Crevecoeur@ugent.be
}

Received 2 November 2011, in final form 9 January 2012

Published 16 March 2012

Online at stacks.iop.org/PMB/57/1963

\begin{abstract}
The simulation of the electroencephalogram (EEG) using a realistic head model needs the correct conductivity values of several tissues. However, these values are not precisely known and have an influence on the accuracy of the EEG source analysis problem. This paper presents a novel numerical methodology for the increase of accuracy of the EEG dipole source localization problem. The presented subspace electrode selection (SES) methodology is able to limit the effect of uncertain conductivity values on the solution of the EEG inverse problem, yielding improved source localization accuracy. We redefine the traditional cost function associated with the EEG inverse problem and introduce a selection procedure of EEG potentials. In each iteration of the presented EEG cost function minimization procedure, potentials are selected that are affected as little as possible by the uncertain conductivity value. Using simulation data, the proposed SES methodology is able to enhance the neural source localization accuracy dependent on the dipole location, the assumed versus actual conductivity and the possible noise in measurements.
\end{abstract}

(Some figures may appear in colour only in the online journal)

\section{Introduction}

For the presurgical evaluation of patients suffering from refractory epilepsy, it is essential to have a correct localization of the epileptogenic zone, i.e. the brain region that is responsible for provoking seizures. Several modalities can be included in this clinical protocol: 
electroencephalogram (EEG), magnetoencephalogram, magnetic resonance imaging (MRI), positron emission tomography, neuropsychological assessments, etc. In particular, the EEG is useful because of its high temporal resolution and by performing EEG source analysis, the epileptogenic zone can be estimated. The estimation of the neural source generators responsible for e.g. epileptic spikes is however subject to some sources of errors: noise in measurements, forward modeling errors and the ill-posedness of the inverse problem.

A first class of model-related errors are source modeling errors. A current-dipole source is suitable because it represents a population of active pyramidal cells at the microscopic level (de Munck et al 1988), but is only valid if the activity itself is limited to a focal region and if it stays focal over a period of time. For patients suffering from epilepsy, focal brain activity is mostly the case. In order to reduce these source modeling errors, it is possible to use more complex source models. Distributed source models can represent an alternative where the inverse problem is highly underdetermined and regularization methods are required, e.g. (Baillet et al 2001). Another source modeling approach consists of limiting the parameters of the multidipolar sources to be less than the number of electrodes e.g. the Recursively Applied and Projected-MUltiple SIgnal Classification (RAP-MUSIC) algorithm (Mosher and Leahy 1999). The information criterion method is a possible means for determining the number of independent sources, as used in Knösche et al (1998) and Bai and He (2006). Steinsträter et al (2010) also investigated the influence of forward modeling errors on EEG beamformers and showed the high sensitivity of the solutions to the conductivity profile in the realistic head model.

A second class of model-related error is the possible inaccurate geometrical modeling of the head (Vanrumste et al 2002). When using patient-specific head models based on T1segmented magnetic resonance images, the geometrical modeling error is limited. A third type of forward modeling errors can be electrode misplacements (Laarne et al 2000). However, by using correct EEG electrode placement techniques, it is possible to decrease that source of error (Sijbers et al 2000), or by using more electrodes in the EEG source analysis problem (Pohlmeier et al 1997). Laarne et al (2000) evaluated the influence of the electrode misplacement and concluded that the use of an incorrect skull conductivity leads to the most serious source of error.

A fourth type of modeling-related errors are head models where the anisotropic behavior of the conductivity is not incorporated. Using diffusion tensor magnetic resonance imaging it is possible to estimate the nerve bundle direction. Haueisen et al (2002), Wolters et al (2006) and Hallez et al (2008) have shown that anisotropically conducting compartments should be incorporated in the volume-conductor head models. Finally, large errors are introduced due to the use of inaccurate absolute conductivity values of several tissues in the volume-conductor head model. The large sensitivity of the EEG measurements to tissue conductivity has been reported e.g. in Haueisen et al (1997), Laarne et al (1999), Gençer and Acar (2004) and Vallaghé and Clerc (2009). The uncertain conductivity values, more specifically the ratio of the skull conductivity to the conductivity values of the soft tissues, have a large influence on the EEG dipole localization accuracy and are the most dominant source of error (Laarne et al 2000, Vanrumste et al 2000, Plis et al 2007, Chen et al 2010). Many values have already been suggested in the literature for the scalp, skull, cerebro-spinal fluid (CSF) and brain: Geddes and Baker (1967) stated that the soft tissue to skull conductivity ratio was 80, Oostendorp et al (2000) 15, Gonçalves et al (2003) 20-50, while Hoekema et al (2003) measured this value to be in the interval 10-40 and Lai et al (2005) as 25. Moreover, the conductivity values may be patient and age dependent; see Gabriel (2005). We assume the conductivity of the soft tissue to be equal to the conductivity of skin, CSF, gray and white matter. It is also hypothesized by Brodbeck et al (2009) that lesions are most likely to change conductivity properties and 
to significantly impair the accuracy of electromagnetic source imaging based on the EEG. Akhtari et al (2010) state that understanding brain electrical conductivity and ways to noninvasively measure them are probably necessary to enhance the ability to localize EEG sources from epilepsy surgery patients.

Plis et al (2007) proposed a probabilistic framework for incorporating the uncertain conductivity values in the reconstruction of neural sources. They concluded that the conductivity of the skull has to be either accurately measured by an independent technique, or that the uncertainties in the conductivity values should be reflected in the source localization estimates. We propose in this paper an alternative solution: a novel technique for limiting the influence of the uncertain conductivity values on the dipole source localization estimates. We have no knowledge of such an already existing technique able to decrease this effect. The presented subspace electrode selection (SES) methodology uses an alternative cost function and employs a selection procedure of the potentials.

\section{Materials and methods}

We use in this study a realistic head model so that in the simulations the impact of the geometrical modeling errors is reduced, and assume the placement of the EEG electrodes and the number of active neural dipoles to be known. We thus assume that the only uncertainty is related to be the conductivity ratio parameter where we aim at reducing its negative impact upon the source localization.

The used head model is patient specific and is based on MR image data. The used head model in the presented simulation study consists of isotropic conductivities where we want to assess the influence of the absolute conductivity values on the recovered neural source locations. When using a realistic head model that incorporates the anisotropy of the conductivity, a closer approximation of reality would be obtained. However, we focus here on providing a proof of concept of the presented SES procedure in a realistic head model with respect to the uncertain skull to soft tissue (skin, cerobrospinal fluid, gray and white matter) conductivity ratio.

In the following we outline the steps for constructing the forward model and in a second stage, we present the inverse techniques for recovering the neural sources.

\subsection{Forward realistic model}

2.1.1. Volume-conductor head model: registration and segmentation. In order to calculate the potentials at the electrodes due to a current-dipole source, a volume-conductor model is needed. The volume-conductor model describes the geometry and the different tissue types. Each tissue type is assigned a label, which is then used to assign a conductivity. In this study we used a realistic head model that was derived from segmented T1-weighted MRI images. The MRI images were obtained by a 3 Tesla MRI scanner (Siemens Trio, Erlangen, Germany) using a three-dimensional MP RAGE sequence with a repetition time (TR) of $1550 \mathrm{~ms}$ and an echo time (TE) of $2.48 \mathrm{~ms}$. The result was a $256 \times 256 \times 176$ matrix of isotropic voxels of $0.9 \mathrm{~mm} \times 0.9 \mathrm{~mm} \times 0.9 \mathrm{~mm}$. To construct the brain compartment, SPM8 was used to segment the white matter, gray matter and CSF (Friston 2007). This resulted in probability values for each voxel, indicating its probability of belonging to white matter, gray matter or CSF. The voxels are then assigned to the compartment for which the voxel had the highest probability. The skull compartment was constructed by a dilation operation of the brain compartment and was on average $6 \mathrm{~mm}$ thick. The scalp compartment was obtained by using opening, closing and hole filling operation on the thresholded MR image. This way we could make a distinction 


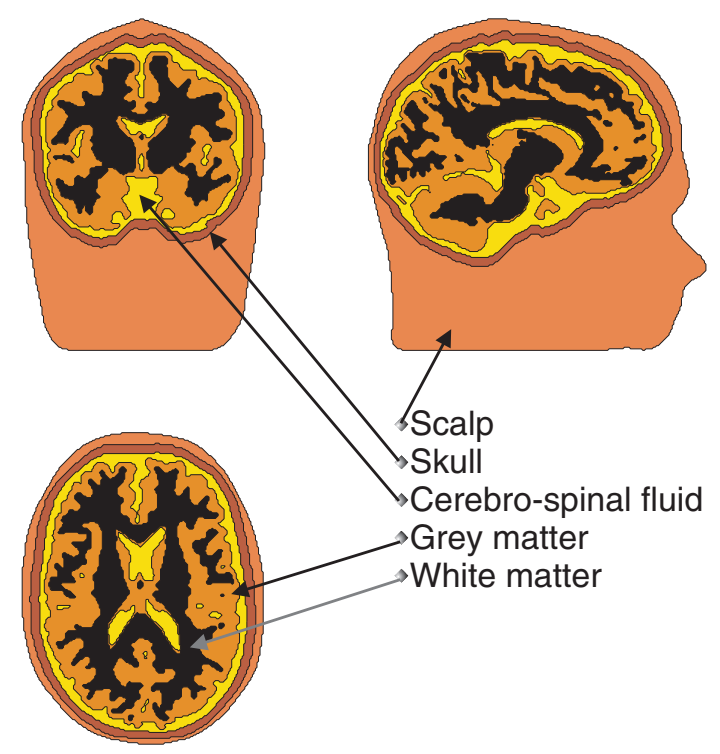

Figure 1. The coronal, sagittal and axial plane of the head model. The test dipoles were placed in each voxel of the gray and white matter. The different compartments are indicated: scalp, skull, CSF, gray matter and white matter.

between head and air. The skull and brain compartments were then added to the whole head model. Figure 1 shows an axial, sagittal and coronal plane of the used volume-conductor head model.

2.1.2. Finite difference method for the forward EEG problem. After defining the head model, the electrode positions have to be determined. We used a 10-10 standard electrode setup, consisting of 81 electrodes. The electrodes were projected onto the scalp.

The forward problem relates the electrode potentials to a dipole source in a specified geometry. This relation can be expressed by solving the Poisson equation.

$$
\nabla \cdot(\sigma(x, y, z) \cdot \nabla \phi(x, y, z))=I \delta\left(\mathbf{r}-\mathbf{r}_{1}\right)-I \delta\left(\mathbf{r}-\mathbf{r}_{2}\right)
$$

with $\phi(x, y, z)$ being the potential distribution inside the head model. $\sigma(x, y, z)$ denotes the conductivity value that is position $(\mathbf{r}=[x, y, z])$ dependent. $\mathbf{r}_{1}, \mathbf{r}_{2}$ are the location coordinates of the monopoles of the dipole: the current source and current sink, respectively. $I$ is the amplitude of the dipole. The numerical method that is used here for solving (1) is based on the finite difference method (FDM), elaborated in Vanrumste et al (2001) and Hallez et al (2005). The method results in the solution of a large sparse linear system of equations that can be solved using the so-called successive overrelaxation method (Barrett et al 1994). Because the computation time would become too expensive when using successive overrelaxation for the solution of the forward problem, the reciprocity theorem is used (Rush and Driscoll 1969). For an extensive validation and further details of the used isotropic forward model, see Vanrumste et al (2001). Figure 2 shows a schematic outline of the steps taken for generating the lead fields using the realistic head model. 


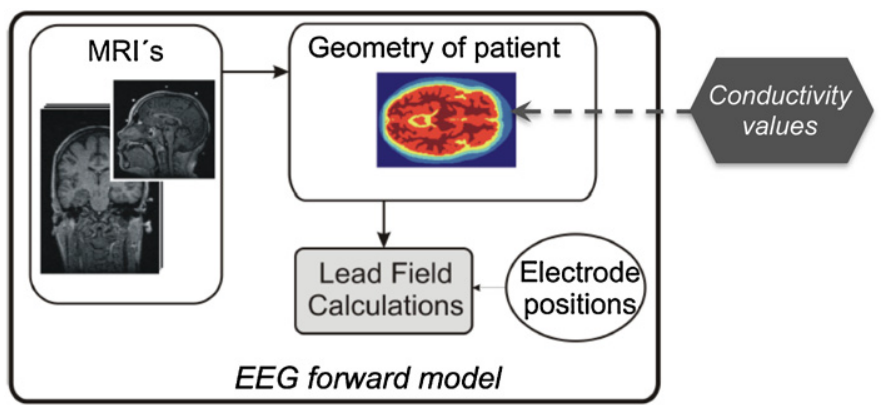

Figure 2. Outline of the forward model: from magnetic resonance images, the geometry of the head is deduced and for given conductivity values and electrode positions, the lead field is calculated.

The so-called lead field matrix $\mathbf{L} \in R^{q \times 3}$ links the dipole location, denoted by $\mathbf{r}_{d}$, with the EEG potentials $v_{m, i}, i=1, \ldots, q$ at the given $q$ electrode positions. We denote the EEG potentials by the $q$-dimensional vector $\mathbf{v}_{m}$, which is linear to the dipole orientation $\mathbf{d}$ :

$$
\mathbf{v}_{m}=\mathbf{L}\left(\mathbf{r}_{d}\right) \mathbf{d} .
$$

2.1.3. Influence of conductivity on the EEG forward problem.. The lead fields $\mathbf{L}\left(\mathbf{r}_{d}\right)$ depend upon the skull to soft tissue conductivity ratio

$$
\xi=\frac{\sigma_{\text {skull }}}{\sigma_{\text {soft }}}
$$

since the calculated potentials $\phi$ in the Poisson equation (1) depend upon $\sigma(x, y, z)$. We denote this dependence by $\mathbf{L}\left(\mathbf{r}_{d}, \xi\right), \mathbf{v}_{m}\left(\mathbf{r}_{d}, \mathbf{d}, \xi\right)$. We assume here the soft tissue to be the brain and the scalp tissue. When assuming a conductivity ratio (e.g. $\widetilde{\xi}=1 / 10)$, which is significantly different from the actual conductivity ratio $\left(\xi_{\text {act }}=1 / 50\right)$ of the patient under study, then a large misfit in the EEG dipole localization can occur because of the effect of $\xi$ on $\mathbf{v}_{m}$.

We can compute the sensitivity of the potentials to the conductivity ratio as follows:

$$
\mathbf{s}\left(\mathbf{r}_{d}, \mathbf{d}, \widetilde{\xi}\right)=\left.\frac{\partial \mathbf{v}_{m}\left(\mathbf{r}_{d}, \mathbf{d}, \xi\right)}{\partial \xi}\right|_{\xi=\widetilde{\xi}}
$$

for a fixed source position and orientation. Some potentials will be highly sensitive to $\xi$, while other potentials are less sensitive since different values $s_{i}(i=1, \ldots, q)$ will be obtained. More elaborated studies of the influence of conductivity on the EEG forward potentials have been carried out by e.g. Haueisen et al (1997) and Laarne et al (1999).

\subsection{Traditional EEG inverse problem}

The EEG inverse problem aims at recovering the neural source locations and orientations that correspond with a certain set of measured EEG potentials $\mathbf{v}_{\text {meas }}$. For clarity, $\mathbf{v}_{m}$ is the set of potentials simulated by the forward solver $\left(v_{m, i}\right.$ is the simulation of the electrode potentials at a certain $i$ th electrode location), while $\mathbf{v}_{\text {meas }}$ is the set of measured potentials ( $v_{\text {meas, } i}$ is the measurement at the $i$ th electrode location). As given previously, vector $\mathbf{v}_{m}$ depends on the dipole location, orientation and conductivity ratio defined by the user, while vector $\mathbf{v}_{\text {meas }}$ arises because of dipole(s) at a certain location, orientation and conductivity ratio that is specific to the patient. 
Several source localization approaches exist, depending on the assumed source model: single-dipole localization based on the minimization of a least-squares cost function (de Munck et al 1988), limited number of multiple dipoles $(<q)$ recovered using multiple signal classification algorithms (Mosher and Leahy 1999) and distributed source models where a highly underdetermined system of linear equations needs to be solved (Pascual-Marqui 1999). In this paper, the nonlinear inverse problem, i.e. nonlinear dependence of scalp potentials $\mathbf{v}_{m}$ with respect to the dipole location(s) $\mathbf{r}_{d}$, is considered. We are not considering distributed source models where the set of scalp potentials is linear to the dipole moments in each source voxel.

The inverse problem for a single dipole is nonlinearly solved by iteratively minimizing a cost function $\mathcal{Y}$

$$
\mathbf{r}_{d}^{*}=\arg \min _{\mathbf{r}_{d}} \mathcal{Y}\left(\mathbf{r}_{d}\right)
$$

which yields recovered dipole location $\mathbf{r}_{d}^{*}$. In (5) a reduction of the number of parameters was performed since it is possible to consider the optimal dipole components in the least-squares sense (see e.g. Baillet et al 2001):

$$
\mathbf{d}_{\text {opt }}=\mathbf{L}^{\dagger} \mathbf{v}_{\text {meas }} \text {. }
$$

$\mathbf{L}^{\dagger}$ is the pseudo inverse of the lead field matrix. For a recovered dipole location $\mathbf{r}_{d}^{*}$, we can calculate the orientation as $\mathbf{d}^{*}=\mathbf{L}\left(\mathbf{r}_{d}^{*}\right)^{\dagger} \mathbf{v}_{\text {meas }}$. $\mathcal{Y}$ is the following least-squares cost function:

$$
\mathcal{Y}\left(\mathbf{r}_{d}\right)=\frac{\left\|\mathbf{v}_{\text {meas }}-\mathbf{L}\left(\mathbf{r}_{d}\right) \mathbf{L}\left(\mathbf{r}_{d}\right)^{\dagger} \mathbf{v}_{\text {meas }}\right\|}{\left\|\mathbf{v}_{\text {meas }}\right\|}
$$

when using the optimal dipole components (6) with $\|\cdot\|$ the $\mathrm{L}_{2}$ norm. For solving (5) the widely used Nelder-Mead simplex is used here as in Huang et al (1998) and Crevecoeur et al (2008). The above inverse problem is formulated for solving the EEG inverse problem at a single time instant and can be extended (for multiple time instances) in a spatio-temporal way.

The solution of the EEG inverse problem for multiple dipoles (with number of dipoles less than the number of channel measurements) can be obtained using the RAP-MUSIC methodology (Mosher and Leahy 1999). We denote here the so-called spatio-temporal data matrix as

$$
\mathbf{F}_{m}=\left[\mathbf{L}\left(\mathbf{r}_{d, 1}\right), \ldots, \mathbf{L}\left(\mathbf{r}_{d, p}\right)\right]\left[\mathbf{D}_{1}^{T}, \ldots, \mathbf{D}_{p}^{T}\right]^{T}
$$

for $p$ dipoles (with locations $\left.\mathbf{r}_{d, i}, i=1, \ldots, p\right)$. The $3 \times n$-matrix $\mathbf{D}_{i}(i=1, \ldots, p)$ represents the time course of the $i$ th dipole moment. $n$ is the number of samples registered by the EEG. The following cost function needs to be maximized for each dipole:

$$
\mathbf{r}_{d, k}^{*}=\arg \max _{\mathbf{r}_{d}}\left(\operatorname{subcorr}\left(\Pi_{\mathbf{A}_{k-1}}^{\perp} \mathbf{L}\left(\mathbf{r}_{d}\right), \Pi_{\mathbf{A}_{k-1}}^{\perp} \Phi_{\text {sig }}\right)_{1}\right),
$$

with $\Pi_{\mathbf{A}_{k-1}}^{\perp}=\left(\mathbf{I}-\mathbf{A}_{k-1} \mathbf{A}_{k-1}^{\dagger}\right)$ being the projection matrix constructed by $\mathbf{A}_{k-1}$, a matrix containing in each column the topographies of the already found $k-1$ sources (Mosher and Leahy 1999). $\Phi_{\text {sig }}$ is the signal subspace of the spatio-temporal measurement matrix. The signal subspace must be compared with the entire span of the gain matrix $\mathbf{L}\left(\mathbf{r}_{d}\right)$, which is possible through the use of principal angles or the cosines of the principal angles (canonical correlations). The principal angles reflect the similarity between the subspaces spanned by the columns of the two matrices. The elements of the subspace correlation vector are ranked in decreasing order. The largest subspace correlation is denoted by $\operatorname{subcorr}(\cdot, \cdot)_{1}$. If $\operatorname{subcorr}\left(\mathbf{S}_{1}, \mathbf{S}_{2}\right)_{1}=1$, then the two subspaces $\mathbf{S}_{1}$ and $\mathbf{S}_{2}$ have at least a one-dimensional subspace in common. If $\operatorname{subcorr}\left(\mathbf{S}_{1}, \mathbf{S}_{2}\right)_{1}=0$, then $\mathbf{S}_{1}$ and $\mathbf{S}_{2}$ are orthogonal. For more details with respect to the $\operatorname{subcorr}(\cdot, \cdot)_{1}$ definition (see Mosher and Leahy 1999). 
The effect of uncertain brain tissue conductivity values onto the inverse solutions can be quantified e.g. using Monte Carlo simulations (Chen et al 2010), stochastic Cramér Rao Bound technique (Plis et al 2007) and polynomial chaos decomposition (Gaignaire et al 2010). We can theoretically express this effect on the inverse solution by redefining (5)

$$
\mathbf{r}_{d}^{*}(\xi)=\arg \min _{\mathbf{r}_{d}} \mathcal{Y}\left(\mathbf{r}_{d}, \xi\right)
$$

where the cost function depends on $\xi$ because $\mathbf{L}\left(\mathbf{r}_{d}, \xi\right)$. The corresponding influence on the dipole orientation can be determined by $\mathbf{d}^{*}=\mathbf{L}\left(\mathbf{r}_{d}^{*}(\xi), \xi\right)^{\dagger} \mathbf{v}_{\text {meas }}$. For the case of recovering a limited number of dipoles, (10) can be iteratively used:

$$
\begin{aligned}
& \mathbf{r}_{d, k}^{*}(\xi)=\arg \min _{\mathbf{r}_{d}} \mathcal{Y}_{k}\left(\mathbf{r}_{d}, \xi\right) \\
& \mathcal{Y}_{k}\left(\mathbf{r}_{d}, \xi\right)=-\operatorname{subcorr}\left(\Pi_{\mathbf{A}_{k-1}}^{\perp} \mathbf{L}\left(\mathbf{r}_{d}, \xi\right), \Pi_{\mathbf{A}_{k-1}}^{\perp} \Phi_{\text {sig }}\right)_{1}
\end{aligned}
$$

for the recovery of the $k=1, \ldots, p$ dipoles.

If one would include the unknown conductivity ratio as a fully nonlinear additional unknown parameter in the cost function, or if one would perform simultaneous optimization over dipole parameters and the conductivity, i.e. solving

$$
\left\{\mathbf{r}_{d}^{*}, \xi^{*}\right\}=\arg \min _{\mathbf{r}_{d}} \mathcal{Y}\left(\mathbf{r}_{d}, \xi\right)
$$

then the localization results would not improve nor would they produce reliable conductivity estimates. This was extensively demonstrated in Plis et al (2007). This approach of extracting the conductivity together with the dipole location was also suggested in Lew et al (2007) and Vallaghé et al (2007).

\subsection{Subspace electrode selection methodology}

The aim of the SES methodology is to reduce the effect of the uncertain $\xi$ values on the inverse solutions, i.e. bringing the recovered $\mathbf{r}_{d}^{*}(\widetilde{\xi})$ with the assumed conductivity ratio $\widetilde{\xi}$ closer to the actual dipole location $\mathbf{r}_{d \text {, act }}$ for a patient with the actual conductivity ratio $\xi_{\text {act }}$ and EEG measurements $\mathbf{v}_{\text {meas }}$. In the notations used below, '*' denotes values that are obtained through numerical inversion, while ' act' is used to denote actual values.

The SES methodology is based on two major ideas: (i) redefinition of the traditional cost function and (ii) selection of potentials in each iteration of the minimization procedure.

2.3.1. Redefinition of the traditional cost functions. In the ideal case (no noise, no electrode mislocations, correct geometry and isotropic conductivities), we can theoretically state that the measured potentials equal the simulated potentials for the actual conductivity ratio $\xi_{\text {act }}$ at the actual dipole position and orientation $\left\{\mathbf{r}_{d, \text { act }}, \mathbf{d}_{\text {act }}\right\}$ :

$$
\begin{aligned}
\mathbf{v}_{\text {meas }} & \equiv \mathbf{v}_{m}\left(\mathbf{r}_{d, \text { act }}, \mathbf{d}_{\text {act }}, \xi_{\text {act }}\right) \\
& \equiv \mathbf{L}\left(\mathbf{r}_{d, \text { act }}, \xi_{\text {act }}\right) \mathbf{d}_{\text {act }} \\
& \equiv \mathbf{L}\left(\mathbf{r}_{d, \text { act }}, \xi_{\text {act }}\right) \mathbf{L}\left(\mathbf{r}_{d, \text { act }}, \xi_{\text {act }}\right)^{\dagger} \mathbf{v}_{\text {meas }},
\end{aligned}
$$

where the optimal dipole components (6) are used to obtain (16). When using however an assumed conductivity ratio $\widetilde{\xi} \neq \xi_{\text {act }}$, then a dipole location $\mathbf{r}_{d}^{*} \neq \mathbf{r}_{d, \text { act }}$ corresponds with the measurement vector:

$$
\mathbf{v}_{\text {meas }} \simeq \mathbf{v}_{m}\left(\mathbf{r}_{d}^{*}, \mathbf{d}^{*}, \widetilde{\xi}\right)
$$


where $\simeq$ is a symbolic notation for the closest $\mathbf{v}_{m}$ to $\mathbf{v}_{\text {meas }}$, meaning that $\left\|\mathbf{v}_{\text {meas }}-\mathbf{v}_{m}\left(\mathbf{r}_{d}^{*}, \mathbf{d}^{*}, \tilde{\xi}\right)\right\|$ is minimal for $\mathbf{r}_{d}^{*}$. $\mathbf{r}_{d}^{*}$ is thus the dipole location obtained when solving the inverse problem; see (5) or (9). So, when recovering a single dipole,

$$
\mathbf{r}_{d}^{*}=\arg \min _{\mathbf{r}_{d}} \frac{\left\|\mathbf{v}_{\text {meas }}-\mathbf{L}\left(\mathbf{r}_{d}, \widetilde{\xi}\right) \mathbf{L}\left(\mathbf{r}_{d}, \widetilde{\xi}\right)^{\dagger} \mathbf{v}_{\text {meas }}\right\|}{\left\|\mathbf{v}_{\text {meas }}\right\|}
$$

may thus differ from the actual dipole location $\mathbf{r}_{d \text {, act }}$ because of noise in the measurement $\mathbf{v}_{\text {meas }}$ or due to the use of wrong $\widetilde{\xi} . \mathbf{r}_{d}$ can be any dipole location in the brain.

The term on the right in (14) can be written as a first-order expansion (up to order $\left.\mathcal{O}\left(\left\|\xi_{\text {act }}-\widetilde{\xi}\right\|^{2}\right)\right)$ :

$$
\mathbf{v}_{\text {meas }}=\mathbf{v}_{m}\left(\mathbf{r}_{d, \text { act }}, \mathbf{d}_{\mathrm{act}}, \tilde{\xi}\right)+\left.\left(\xi_{\mathrm{act}}-\tilde{\xi}\right) \frac{\partial \mathbf{v}_{m}\left(\mathbf{r}_{d, \mathrm{act}}, \mathbf{d}_{\mathrm{act}}, \xi\right)}{\partial \xi}\right|_{\xi=\widetilde{\xi}},
$$

where the second right-hand side term depends on the effect of the uncertainty on the forward problem; see equation (4). If one uses a certain assumed conductivity ratio $\widetilde{\xi}$, then (19) would be a sufficient forward model, but $\xi_{\text {act }}$ is however unknown and thus the Taylor coefficient. More concretely, if one patient's EEG is measured and its conductivity ratio is $\xi_{\text {act }}$ (unknown), while the one that is used in the modeling is $\widetilde{\xi}$, then we use a wrong forward model $\mathbf{v}_{m}\left(\mathbf{r}_{d}, \mathbf{d}, \widetilde{\xi}\right)$. The linear model (19) is a more correct approximation of the true model where the Taylor coefficient is estimated using the methods described here below.

The Taylor coefficient $\xi_{\text {act }}-\widetilde{\xi}$ can be approximated by fitting the data set

$$
\mathbf{y}=\mathbf{v}_{\text {meas }}-\mathbf{v}_{m}\left(\mathbf{r}_{d}, \mathbf{d}, \tilde{\xi}\right)
$$

with

$$
\mathbf{s}=\left.\frac{\partial \mathbf{v}_{m}\left(\mathbf{r}_{d}, \mathbf{d}, \xi\right)}{\partial \xi}\right|_{\xi=\tilde{\xi}}
$$

defined by

$$
\alpha^{*}=\arg \min _{\alpha}\|\mathbf{y}-\alpha \mathbf{s}\| .
$$

But since in the iterative minimization, $\mathbf{r}_{d} \neq \mathbf{r}_{d \text {, act }}$, this fitting is not linear so that errors are made with respect to the fitting (i.e. $\alpha^{*}$ is different from the correct $\xi_{\text {act }}-\widetilde{\xi}$ ) but when $\mathbf{r}_{d}$ approaches $\mathbf{r}_{d \text {, act }}$ this Taylor coefficient is better determined.

Figures 3-6 illustrate the impact of the fitting constant on the SES cost function. We start from 'measured' potentials $\mathbf{v}_{\text {meas }}$ that are generated with actual $\xi_{\text {act }}$ and actual dipole locations $\mathbf{r}_{d \text {, act }}=\left[x_{d, \text { act }}, y_{d, \text { act }}, z_{d \text {,act }}\right]$. We look closer to the fitting (22) as a function of $\mathbf{r}_{d}$ (see dependence in vectors $\mathbf{y}$ and $\mathbf{s}$ in equations (20) and (21), respectively) for a certain fixed assumed conductivity ratio $\widetilde{\xi}$.

Figure 3 depicts the percentage error of fitting constant $\alpha^{*}$ compared to the true $\xi_{\text {act }}-\widetilde{\xi}$ :

$$
E_{\text {fit }}=\frac{\alpha^{*}-\left(\xi_{\text {act }}-\tilde{\xi}\right)}{\xi_{\text {act }}-\tilde{\xi}} \times 100 \%
$$

is depicted for varying $\mathbf{r}_{d}-\mathbf{r}_{d \text {,act }}$. From this figure, we can observe that the fitting constant can significantly differ from the true $\xi_{\text {act }}-\widetilde{\xi}$. The root-mean-square error between the fitted data sets $\mathbf{y}$ and $\alpha^{*} \mathbf{s}$ is also rising for $\mathbf{r}_{d}$ further away from $\mathbf{r}_{d \text {, act }}$; see figure 4 .

It is important to determine the impact of wrongly estimated fit constants $\alpha$ onto the linear model or onto the cost function

$$
\frac{\left\|\mathbf{v}_{\text {meas }}-\left(\mathbf{v}_{m}\left(\mathbf{r}_{d}, \mathbf{d}, \tilde{\xi}\right)+\alpha \mathbf{s}\right)\right\|}{\left\|\mathbf{v}_{\text {meas }}\right\|}
$$




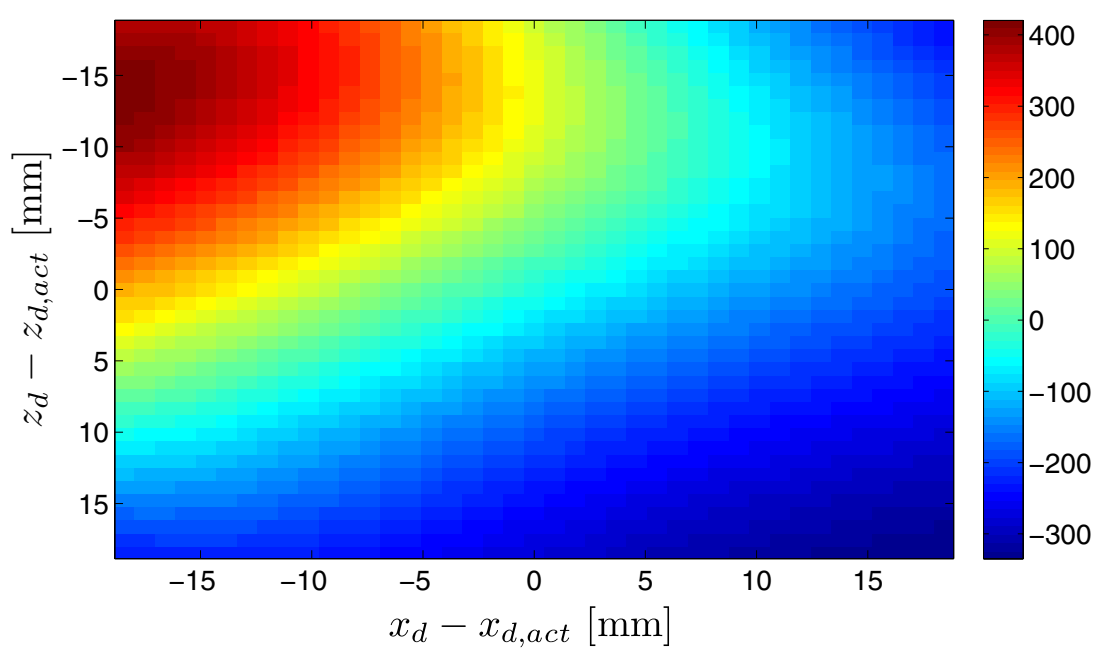

Figure 3. Percentage error $E_{\mathrm{fit}}(\%)$ of fitting constant $\alpha$ and $\mathbf{r}_{d}-\mathbf{r}_{d \text {, act }}$ in a two-dimensional (2D) slice for a certain constant $y_{d} \equiv y_{d \text {,act }} \cdot \xi_{\text {act }}-\widetilde{\xi}$ is 0.0259 in this simulation study.

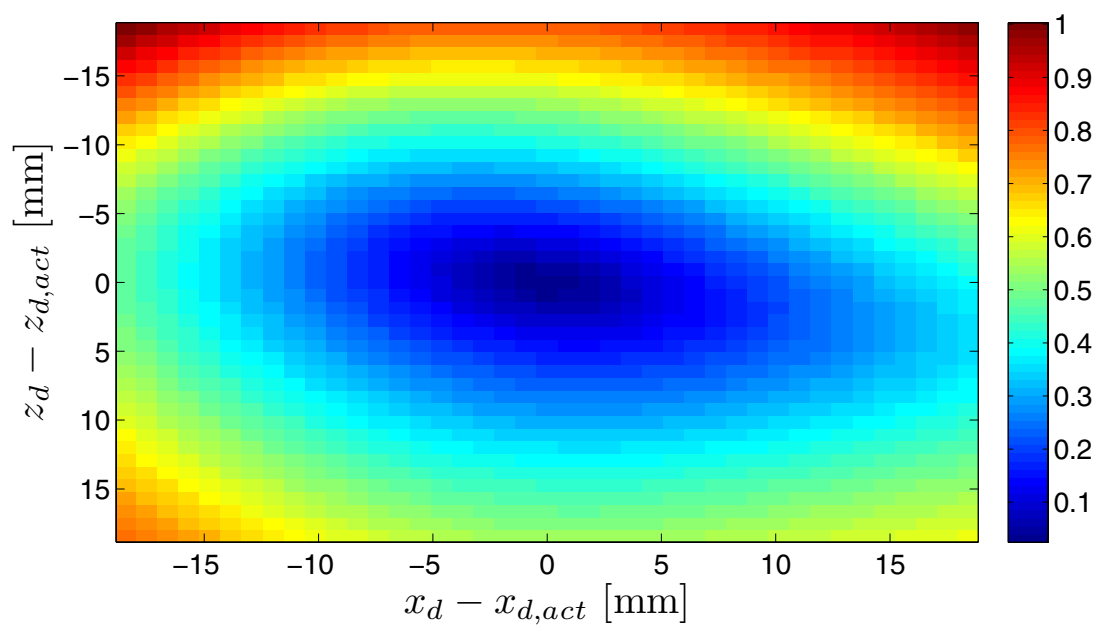

Figure 4. Root mean square error between fitted data sets $\mathbf{y}$ and $\alpha^{*} \mathbf{s}$ in a 2D slice for a certain constant $y_{d} \equiv y_{d, \text { act }}$. The residual error corresponds with the $\alpha$ fitted from figure 3 .

and compare this with the cost function with true $\alpha=\xi_{\text {act }}-\tilde{\xi}$. Figures 5 and 6 , respectively, show the cost function surface when using the wrongly fitted $\alpha$ coefficients and when using the true $\alpha=\xi_{\text {act }}-\widetilde{\xi}$. It is clear from these figures that even an $E_{\text {fit }}$ of $100 \%-400 \%$ yields a cost that is high enough. So, the further away $\mathbf{r}_{d}$ from $\mathbf{r}_{d \text {, act }}$, the higher the error between the fitted $\alpha$ and true $\xi_{\text {act }}-\widetilde{\xi}$ but also the higher the cost function error (even for wrongly fitted coefficients). This is because the zeroth-order term in the linear model has a large impact compared to the first-order term onto the cost function.

Figure 7 illustrates equation (19) with potential values for actual $\xi_{\text {act }}=0.0422$ and estimated potential values using the linear model with assumed $\widetilde{\xi}=0.025$ and estimated 


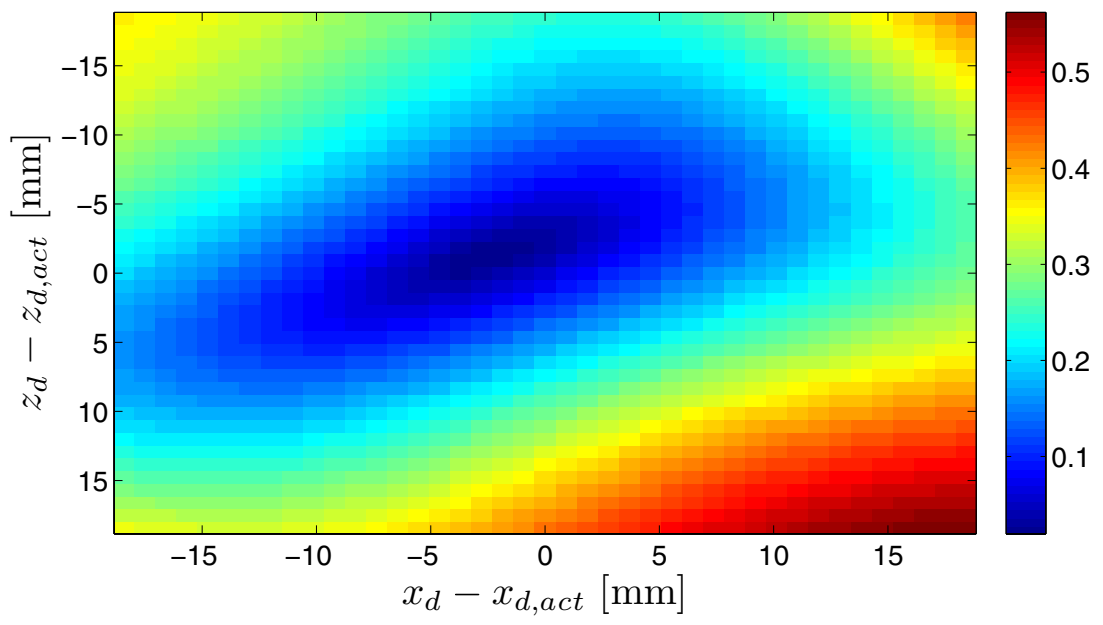

Figure 5. SES-based cost function with fitted $\alpha$ constants (see figure 3 ) in a 2D slice for a certain constant $y_{d} \equiv y_{d \text {,act }}$. The $\alpha$ corresponds with the one that was calculated for figure 3 .

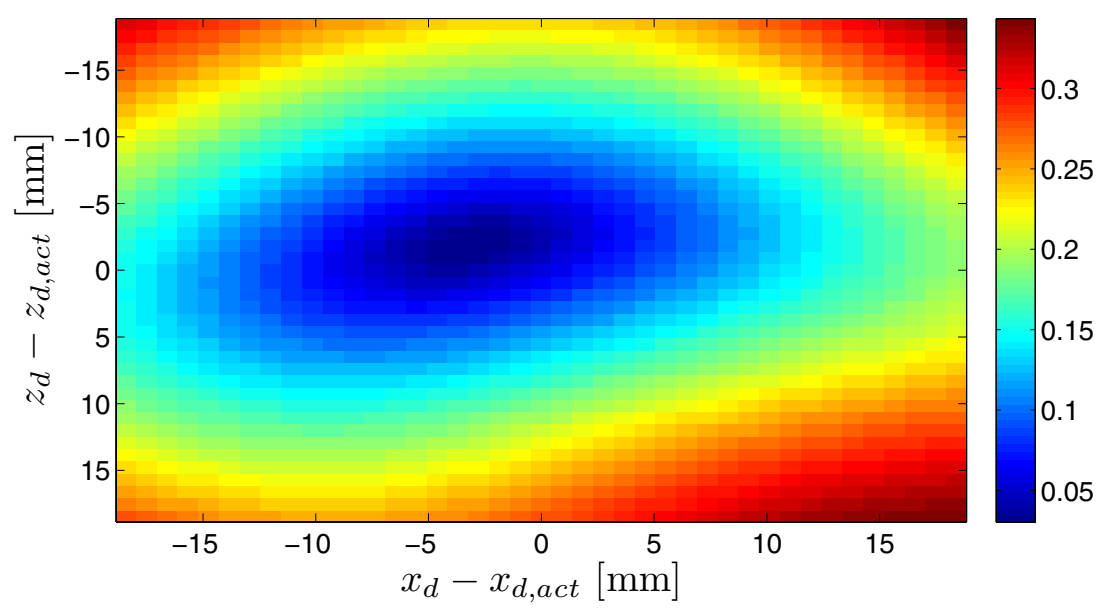

Figure 6. SES-based cost function with true $\alpha=\xi_{\text {act }}-\tilde{\xi}$ constants in a 2D slice for a certain constant $y_{d} \equiv y_{d, \text { act }}$.

potential values using the model $\mathbf{v}_{m}\left(\mathbf{r}_{d, \text { act }}, \mathbf{d}_{\text {act }}, \tilde{\xi}\right)$. The Taylor coefficient was determined using the above-mentioned fitting procedure. We can observe that the estimated potentials based on the linear model are a closer approximation of the actual potentials, compared to the estimated potentials based on $\mathbf{v}_{m}\left(\mathbf{r}_{d \text {, act }}, \mathbf{d}_{\text {act }}, \widetilde{\xi}\right)$ versus the actual potentials.

It is also possible to write (14) as a second-order expansion

$$
\begin{gathered}
\mathbf{v}_{\text {meas }}=\mathbf{v}_{m}\left(\mathbf{r}_{d, \text { act }}, \mathbf{d}_{\text {act }}, \tilde{\xi}\right)+\left.\left(\xi_{\text {act }}-\widetilde{\xi}\right) \frac{\partial \mathbf{v}_{m}\left(\mathbf{r}_{d, \text { act }}, \mathbf{d}_{\text {act }}, \xi\right)}{\partial \xi}\right|_{\xi=\widetilde{\xi}} \\
+\left.\frac{\left(\xi_{\text {act }}-\widetilde{\xi}\right)^{2}}{2} \frac{\partial^{2} \mathbf{v}_{m}\left(\mathbf{r}_{d, \text { act }}, \mathbf{d}_{\mathrm{act}}, \xi\right)}{\partial^{2} \xi}\right|_{\xi=\tilde{\xi}}
\end{gathered}
$$




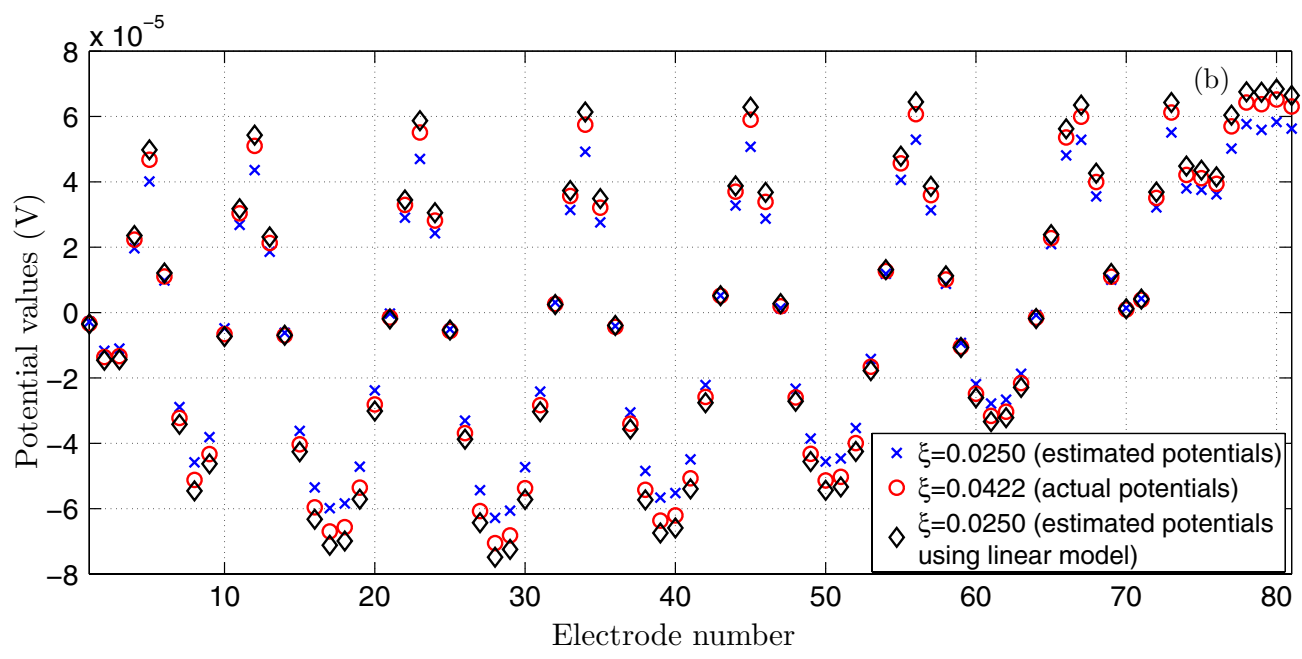

Figure 7. Calculated EEG potentials for $\xi=0.0422$ using $\mathbf{L}\left(\mathbf{r}_{d}, \xi\right) \mathbf{d}$ that corresponds with the EEG potentials (actual $\xi=0.0422$ ), estimated EEG potentials using $\xi=0.0250$ and estimated potentials using linear model (19) for assumed conductivity ratio $\xi=0.0250$.

Here, $\alpha$ can be determined by performing the following:

$$
\alpha^{*}=\arg \min _{\alpha}\left\|\mathbf{y}-\alpha \mathbf{s}-\alpha^{2} \mathbf{h}\right\|
$$

with $\mathbf{h}=\left.\frac{1}{2} \frac{\partial^{2} \mathbf{v}_{m}\left(\mathbf{r}_{d, a c t}, \mathbf{d}_{\text {act }}, \xi\right)}{\partial^{2} \xi}\right|_{\xi=\widetilde{\xi}}$.

A different fitting procedure needs to be performed when a limited number of multiple dipoles need to be recovered. Indeed, the cost function (12) has no longer an $m$-dimensional measurement vector as input but an $m \times r$-dimensional signal subspace matrix with $r$ being the number of topographies in the signal subspace. A different sensitivity than (4) needs to be defined. We consider in the spatio-temporal case the use of the principal vector $\mathbf{u}$ that is associated with the principal angle of the subspace correlation function. This principal vector can be calculated following Golub and Loan (1996) and depends in the $l$ th iteration of the minimization procedure (11) of the $k$ th dipole upon $\Phi_{\text {sig }}$ and the conductivity dependent $\mathbf{L}\left(\mathbf{r}_{d}, \xi\right)$. Therefore, the following sensitivity to the conductivity ratio is calculated in each iteration:

$$
\mathbf{s}_{\mathrm{sub}}=\left.\frac{\partial \mathbf{u}}{\partial \xi}\right|_{\widetilde{\xi}}
$$

and can be approximated by finite differentiation. $\alpha$ is now approximated by fitting $\mathbf{u}$ with $\mathbf{S}_{\text {sub }}$.

Using the above, we are able to adapt the traditional cost functions for single-dipole localization (7) and for multiple-dipole localization (12): $\mathcal{Y} \rightarrow \mathcal{Y}^{\mathrm{SES}}$. The following basic operation needs to be performed in these traditional cost functions:

$$
\mathbf{L}\left(\mathbf{r}_{d}, \widetilde{\xi}\right) \rightarrow \mathbf{L}\left(\mathbf{r}_{d}, \widetilde{\xi}\right)+\alpha \mathbf{T}
$$

for the first-order case with the above-mentioned fitting procedures. $\mathbf{T}$ is similar to $\mathbf{S}$

$$
T_{i j}=\frac{\partial L_{i j}}{\partial \xi}, \quad i=1, \ldots, q ; \quad j=1, \ldots, 3,
$$


i.e. $\mathbf{S}$ is the derivative of the potential values to the conductivity ratio, while matrix $\mathbf{T}$ is the derivative of the lead field matrix to the conductivity ratio. Cost function (7) becomes

$$
\mathcal{Y}^{\mathrm{SES}}\left(\mathbf{r}_{d}, \xi\right)=\frac{\left\|\mathbf{v}_{\text {meas }}-\left(\mathbf{L}\left(\mathbf{r}_{d}, \xi\right)+\alpha \mathbf{T}\right)\left(\mathbf{L}\left(\mathbf{r}_{d}, \xi\right)+\alpha \mathbf{T}\right)^{\dagger} \mathbf{v}_{\text {meas }}\right\|}{\left\|\mathbf{v}_{\text {meas }}\right\|}
$$

and (12) becomes

$$
\mathcal{Y}_{k}^{\mathrm{SES}}\left(\mathbf{r}_{d}, \xi\right)=-\operatorname{subcorr}\left(\Pi_{\mathbf{A}_{k-1}}^{\perp} \mathbf{L}\left(\mathbf{r}_{d}, \xi\right)+\alpha \mathbf{T}, \Pi_{\mathbf{A}_{k-1}}^{\perp} \Phi_{\text {sig }}\right)_{1} .
$$

2.3.2. Selection of electrodes. During the minimization (10) or (11), iterations $\mathbf{r}_{d}^{(l)}$ are evaluated in the forward model and due to the uncertain conductivity values, the minimization path is affected by the uncertainty. Therefore, we select in the $l$ th iteration those potentials with the lowest sensitivity to conductivity. Potentials with the highest sensitivity are eliminated in that iteration since they carry information that is affected by the uncertain conductivity. There are two possibilities to select the potentials: either select the potentials on the basis of a threshold: $s_{i}<\epsilon$ with $\epsilon$ being a predefined threshold value or select each time a fixed limited number of potentials. At each evaluation of the forward model, the following selection operation is carried out upon the data sets:

$$
\begin{aligned}
& \mathbf{v}_{\text {meas }} \in R^{m} \rightarrow \mathbf{v}_{\text {meas }}^{S} \in R^{s} \\
& \Phi_{\text {sig }} \in R^{m \times r} \rightarrow \Phi_{\text {sig }}^{S} \in R^{s \times r} \\
& \mathbf{v}_{m} \in R^{m} \rightarrow \mathbf{v}_{m}^{S} \in R^{s} \\
& \mathbf{L} \in R^{m \times 3} \rightarrow \mathbf{L}^{S} \in R^{s \times 3} \\
& \mathbf{T} \in R^{m \times 3} \rightarrow \mathbf{T}^{S} \in R^{s \times 3}
\end{aligned}
$$

with $s<q$ being the number of selected electrodes out of the total of $q$ electrodes. Since the sensitivity is taken into account in the cost function, selection is also carried out on the sensitivity matrices. Taking operations (32) and (28) into account, the SES cost functions $\mathcal{Y}^{\mathrm{SES}}\left(\mathbf{r}_{d}, \xi\right)$ in (30) and (31) become

$$
\frac{\left\|\mathbf{v}_{\text {meas }}^{S}-\left(\mathbf{L}^{S}\left(\mathbf{r}_{d}, \xi\right)+\alpha \mathbf{T}^{S}\right)\left(\mathbf{L}^{S}\left(\mathbf{r}_{d}, \xi\right)+\alpha \mathbf{T}^{S}\right)^{\dagger} \mathbf{v}_{\text {meas }}^{S}\right\|}{\left\|\mathbf{v}_{\text {meas }}^{S}\right\|}
$$

and

$$
-\operatorname{subcorr}\left(\Pi_{\mathbf{A}_{k-1}}^{\perp} \mathbf{L}^{S}\left(\mathbf{r}_{d}, \xi\right)+\alpha \mathbf{T}^{S}, \Pi_{\mathbf{A}_{k-1}}^{\perp} \Phi_{\text {sig }}^{S}\right)_{1},
$$

respectively. The above can be extended to a second-order SES cost function.

Figure 8 outlines the SES methodology: in a first stage the potentials and sensitivities are calculated. In a second stage, selection is carried out on the calculated and measured EEG data set with the internal fitting procedure. Thirdly, using this data set, the SES cost functions (37) and (38) are calculated leading to an update of the dipole parameters. The update can be carried out using a given minimization procedure. Here, the Nelder-Mead simplex method is used. The 'subspace' in the SES method refers to the use of a subset of electrodes that are used in the iterative procedure: i.e. the set of measured potentials used in the iterative procedure: $\mathbf{v}_{\text {meas }}^{S} \subseteq \mathbf{v}_{\text {meas }}$ and the set of simulated potentials $\mathbf{v}_{m}^{S} \subseteq \mathbf{v}_{m}$ (or lead field operators). The used subset of potentials or subspace can vary iteratively. 


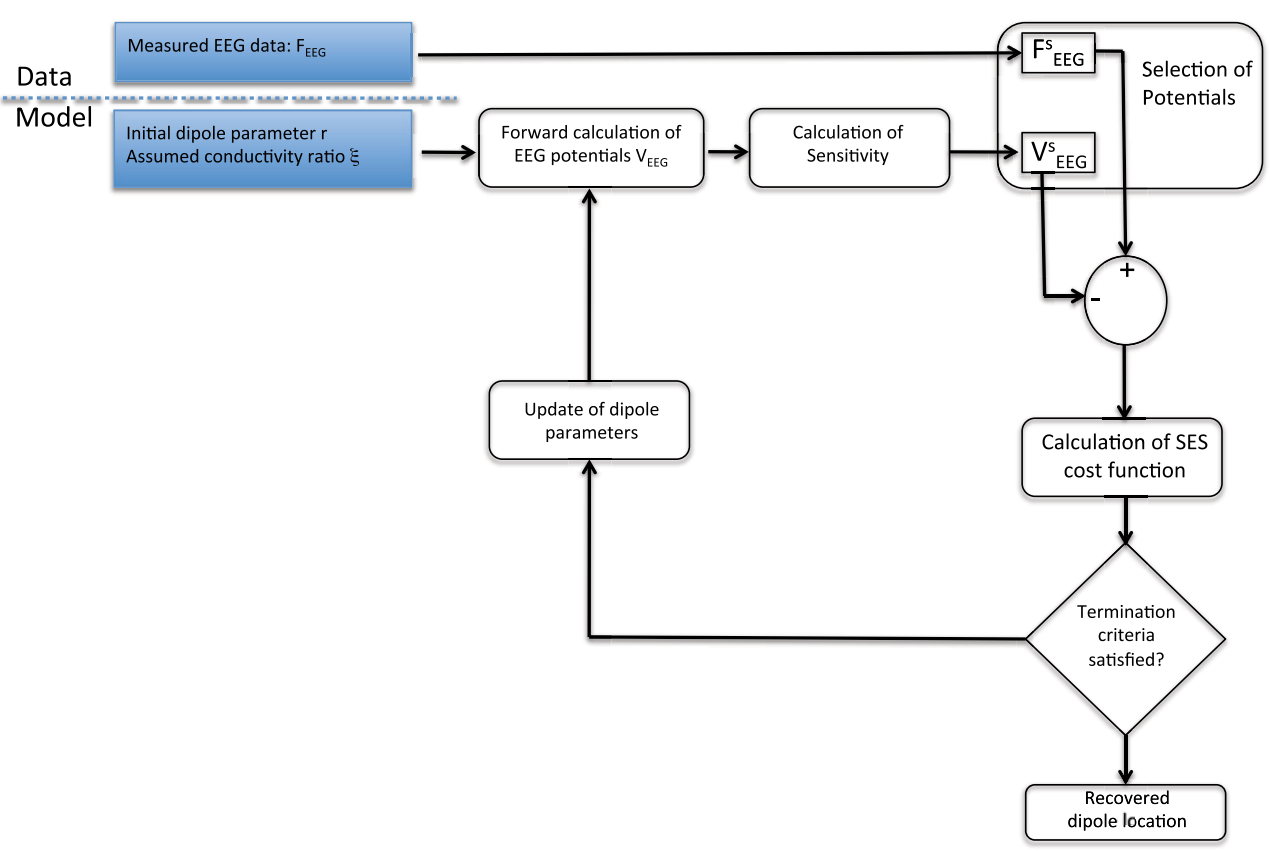

Figure 8. Schematic overview of the iterative SES methodology.

If one used electrode selection with the traditional cost function, then the same results would be obtained. Indeed, in the theoretical limit $k \rightarrow \infty$, the traditional solutions given by equation (5) (with (7))

$$
\mathbf{r}_{d}^{*}=\arg \min _{\mathbf{r}_{d}} \frac{\left\|\mathbf{v}_{\text {meas }}-\mathbf{L}\left(\mathbf{r}_{d}\right) \mathbf{L}\left(\mathbf{r}_{d}\right)^{\dagger} \mathbf{v}_{\text {meas }}\right\|}{\left\|\mathbf{v}_{\text {meas }}\right\|}
$$

would be the same as the solutions obtained with selection (with same formulation of the cost function)

$$
\mathbf{r}_{d}^{*, S}=\arg \min _{\mathbf{r}_{d}} \frac{\left\|\mathbf{v}_{\text {meas }}^{S}-\mathbf{L}^{S}\left(\mathbf{r}_{d}\right) \mathbf{L}^{S}\left(\mathbf{r}_{d}\right)^{\dagger} \mathbf{v}_{\text {meas }}^{S}\right\|}{\left\|\mathbf{v}_{\text {meas }}^{S}\right\|} .
$$

Indeed, the potentials corresponding to the recovered $\mathbf{r}_{d}^{*}$ approximate best the measured potentials $\mathbf{v}_{\text {meas }}$, while the potentials corresponding to $\mathbf{r}_{d}^{*, S}$ also approximate best the subset selected measured potentials $\mathbf{v}_{\text {meas }}^{S} \cdot \mathbf{v}_{\text {meas }}^{S}$ is a subset of $\mathbf{v}_{\text {meas }}$. That was the original reason for modifying the cost function. When using the modified cost function (30), different dipole locations will be recovered than (39) and are closer to the actual dipole locations because the modified forward model based on (19) is a better approximation of reality. Moreover, when using selection with cost function (37), the recovered dipole locations will be even closer to the actual dipole locations because the $\alpha$ coefficient in (37) is better determined (i.e. less affected by the uncertain conductivity ratio) than the one in (30).

\section{Results and discussion}

The methodology presented in this paper is dedicated to the accurate localization of focal epileptic zones. These neural sources can be represented by one or multiple dipoles where 


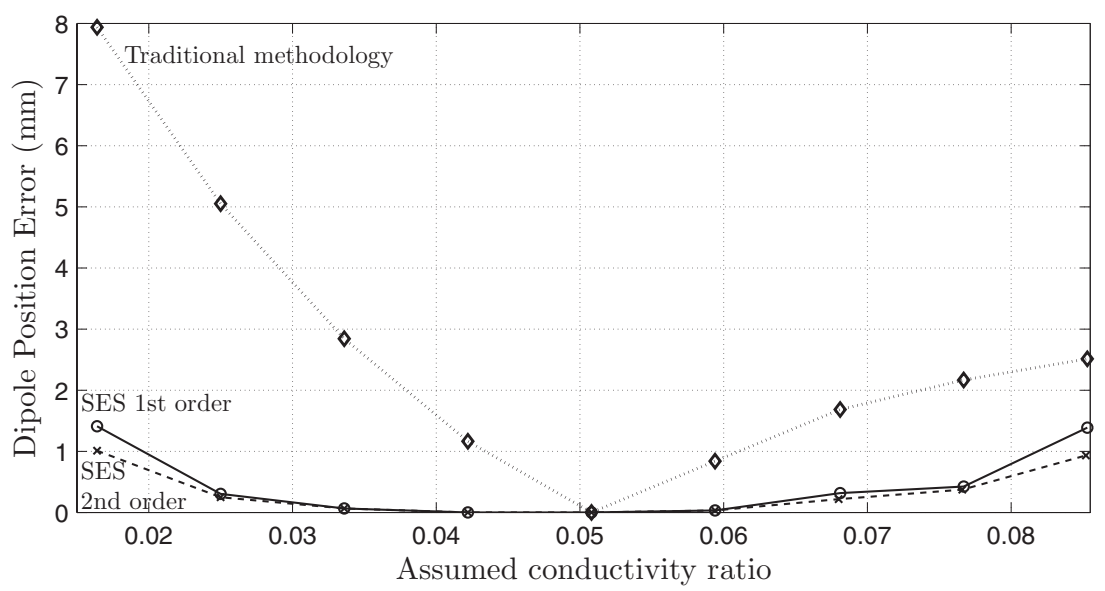

Figure 9. Dipole position error of a single dipole when using traditional method and selection methodology $(s=20)$ of the first and second order. The dipole is located in the hippocampus.

the EEG inverse problem is nonlinear. The accuracy is of clinical importance since resection of the epileptic zone follows the presurgical evaluation. The extension of the methodology toward linear EEG inverse methodologies is not addressed in this paper.

Note that the used nonlinear dipole reconstruction methods are known to have poor performance with respect to multiple dipoles (Pascual-Marqui 1999) and that subspace source localization (RAP-MUSIC) methods have poor performance with respect to correlated sources (Liu and Schimpf 2006). The SES methodology based on the nonlinear cost function (30) and the subspace cost function (31) will have the same behavior, respectively.

\subsection{Single-dipole localization}

3.1.1. No noise case.. In order to investigate the influence of assuming an incorrect conductivity, we first solve the forward problem using actual dipole specifications $\mathbf{r}_{d \text {, act }}, \mathbf{d}_{\text {act }}$ for a certain actual conductivity ratio $\xi_{\text {act }}$. Starting from these potentials $\mathbf{v}_{m}\left(\mathbf{r}_{d, \text { act }}, \mathbf{d}_{\text {act }}, \xi_{\text {act }}\right)$ (without adding noise), we solve the inverse problem using a forward model with assumed conductivity ratio $\widetilde{\xi}$. We evaluate the dipole position error using the Euclidean distance between the original dipole location $\mathbf{r}_{d \text {, act }}$ and the estimated dipole location $\mathbf{r}_{d}^{*}:\left\|\mathbf{r}_{d}^{*}-\mathbf{r}_{d \text {, act }}\right\|$. Since we are using a numerical realistic head model, the lead field is not a continuous function of $\xi$ but needs to be calculated for different discrete conductivity ratio values. The sensitivity $\mathbf{s}$ and second-order term $\mathbf{h}$ are approximated using finite differentiation of these lead fields to the conductivity ratio. Lead field matrices were calculated for the following values of $\xi$ : $0.0164+n 0.0086$ for $n=0, \ldots, 8$. In this way, we cover the interval $1 / 60$ until $1 / 10$. The values in this interval are usually used as possible values for the conductivity ratio.

Figure 9 shows the single-dipole localization error where the actual conductivity ratio is assumed to be around $\xi_{\text {act }}=1 / 20$. We observe from this figure that the SES methodology decreases the dipole position error. Indeed, when assuming a conductivity value $\widetilde{\xi}=1 / 60$, the error is decreased from 8 to $1.5 \mathrm{~mm}$ when using the first-order SES methodology. The dipole position error is even more decreased when using the second-order SES, especially for assumed conductivity ratios far from the actual conductivity ratio. This can be explained by the fact that the validity region of the linear first-order model is smaller than the 
second-order model. The impact of the fitted Taylor $\alpha$ coefficients (that may differ from $\xi_{\text {act }}-\widetilde{\xi}$; see figure 3 ) onto the recovered solutions can be observed in figure 9 since there is still a dipole position error.

In a next stage, we investigated the global efficiency of the methodology. We placed in each voxel of the gray matter a certain dipole and calculated its corresponding EEG potential for $\xi_{\text {act }}=1 / 20$. The inverse problem was then solved using an assumed conductivity ratio of $\widetilde{\xi}=1 / 40$. The dipole position errors in the axial plane are shown in figures 10 (a)-(c) for dipoles oriented in the $x, y$ and $z$ directions, respectively. In these figures, the $x(y)$-axis is the horizontal (vertical) axis $((x, y)$-plane defines the transverse plane of the geometry, $(y, z)$ defines the coronal plane and $(x, z)$ defines the sagittal plane).

We observe an overall decrease in dipole position error where errors of up to $1 \mathrm{~cm}$ are decreased to less than $4 \mathrm{~mm}$. Figures 11(a)-(c) illustrate the corresponding dipole orientation errors. The angle between the vector components of the original dipole $\mathbf{d}_{\text {act }}$ and estimated dipole orientation $\mathbf{d}^{*}$ is calculated using the cosine rule

$$
\angle\left(\mathbf{d}^{*}, \mathbf{d}_{\mathrm{act}}\right)=\arccos \left(\frac{\mathbf{d}^{*} \mathbf{d}_{\mathrm{act}}}{\left\|\mathbf{d}^{*}\right\|\left\|\mathbf{d}_{\mathrm{act}}\right\|}\right) .
$$

3.1.2. Noise case. Contrary to the previous simulation study, we added noise $\mathbf{n}$ to the previously calculated potentials: $\mathbf{v}_{m}\left(\mathbf{r}_{d, \text { act }}, \mathbf{d}_{\text {act }}, \xi_{\text {act }}\right)+\mathbf{n}$. The noise is white Gaussian with a certain standard deviation $\sigma_{n}$. The noise level in the data set is determined by $\frac{\sigma_{n}}{v_{m, \text { RMS }}}$ with $v_{m \text {, RMS }}$ being the root-mean-square value of the no noise simulated potentials $\mathbf{v}_{m}\left(\mathbf{r}_{d, \text { act }}, \mathbf{d}_{\text {act }}, \xi_{\text {act }}\right)$. In order to determine in an average way the influence of noise at a certain noise level, the inverse problem is solved 50 times with random noise added to the potentials.

Figure 12 shows the average dipole position error for various noise levels when using the traditional methodology and when using the SES methodology with fitting procedure based on $\mathbf{s}$ and $\mathbf{y}$, as mentioned in section 2.3.1. It is clear that the advantage of SES is counteracted by the noise. The reason for the bad noise robustness of this procedure is because the residual of the fitting becomes very high when noise is available in the measurements, i.e. the cost function is no longer correct. For very high noise levels, the SES methodology is even worse than the traditional methodology. Therefore, we used instead the fitting procedure based on the principal vector of the subspace correlation function:

$$
\operatorname{subcorr}\left(\mathbf{L}\left(\mathbf{r}_{d}, \xi\right), \mathbf{v}_{\text {meas }}\right)_{1} .
$$

A more accurate fit was achieved since the principal vector is much less affected by the noise than the absolute values of the calculated potentials. Indeed, figure 13 shows the average dipole position error when using the traditional cost function and when using the SES methodology with fitting of data sets $\mathbf{u}$ and $\mathbf{s}_{\text {sub }}$.

The reason why the methodology is failing for higher noise levels (figure 12) when using the nonlinear cost function (30) is twofold. Information about the dipole positions and strength that hold important information can be eliminated in the selection procedure. But this is not the only reason since the subspace-based SES cost function is robust to noise as can be observed in figure 13 when reconstructing the same dipole as in figure 12. The second reason is that the fitting procedure based on $\mathbf{y}$ and $\mathbf{s}$ sets (for cost function (30)) is not so robust to noise as when the fitting is performed on sets $\mathbf{u}$ and $\mathbf{s}_{\text {sub }}$ (for cost function (31)). This is illustrated by figure 14 where the $E_{\text {fit }}$ is shown in the case of noise with noise level 0.2 .

We can observe that the SES of second order has little advantage compared to the SES of first order when noise is included in the measurements. This result shows that it is possible to use the SES methodology in the realistic noise case, provided that a correct internal fitting 

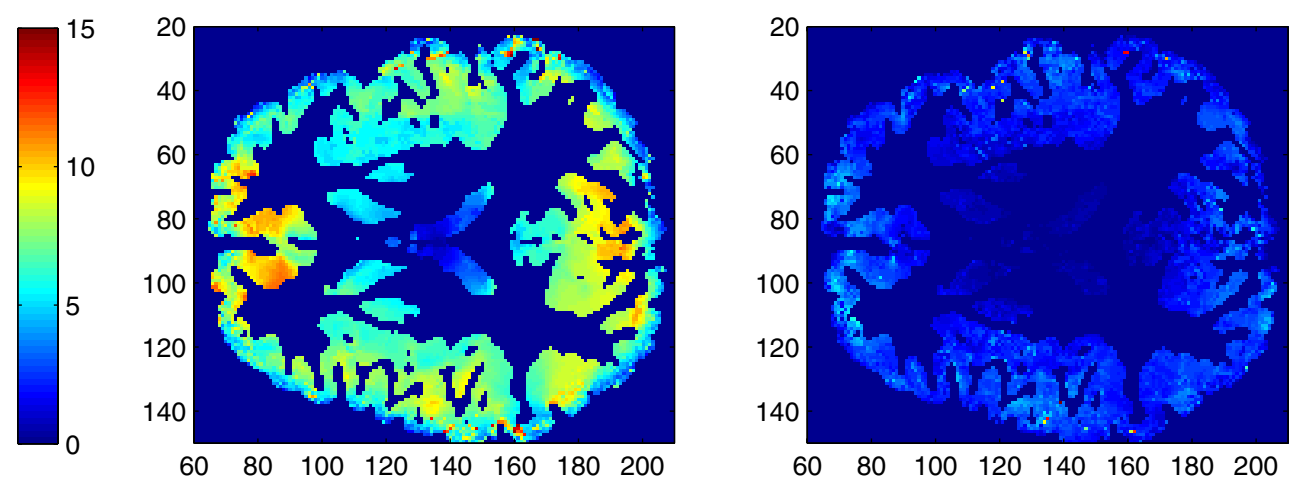

(a) Dipole position errors ( $\mathrm{mm}$ ) of dipoles oriented in the x-direction when using traditional method (left) and when using Subspace Electrode Selection (right).
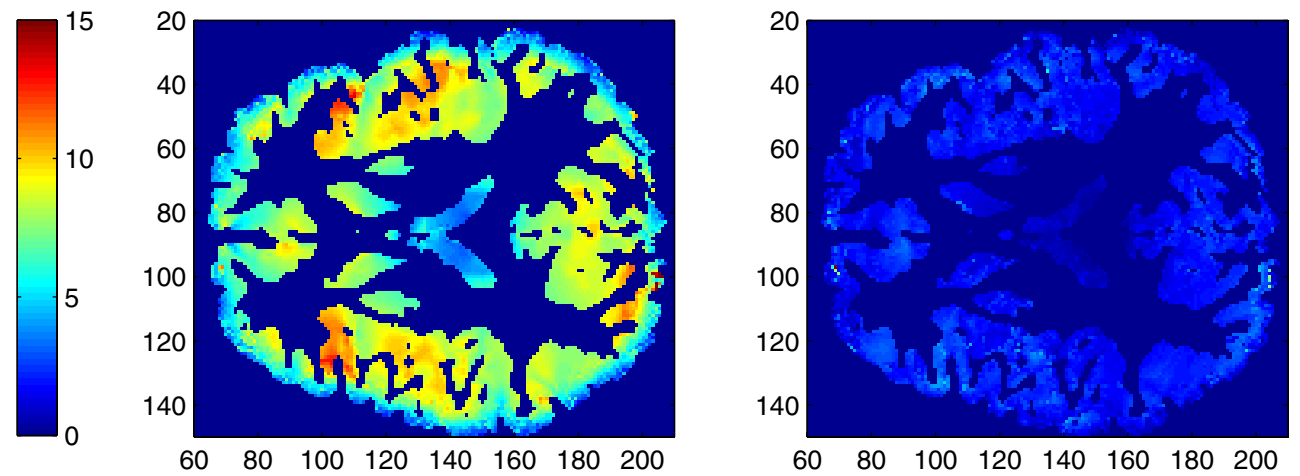

(b) Dipole position errors ( $\mathrm{mm}$ ) of dipoles oriented in the y-direction when using traditional method (left) and when using Subspace Electrode Selection (right).
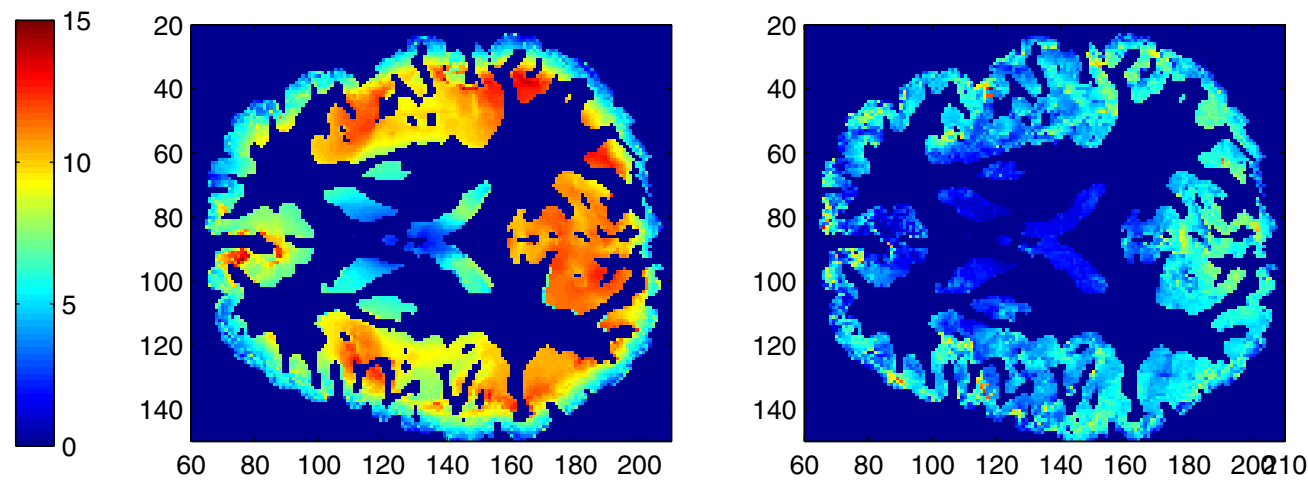

(c) Dipole position errors ( $\mathrm{mm}$ ) of dipoles oriented in the z-direction when using traditional method (left) and when using Subspace Electrode Selection (right).

Figure 10. Dipole position errors for (a) $x$-directed dipoles, (b) $y$-directed dipoles and (c) $z$-directed dipoles. 

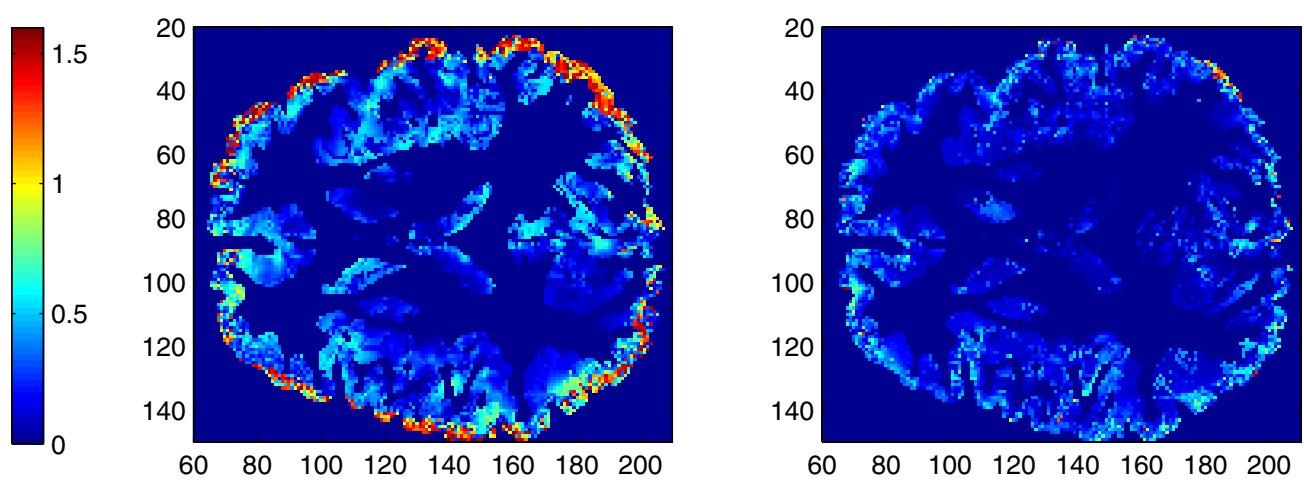

(a) Dipole orientation errors of dipoles oriented in the x-direction when using traditional method (left) and when using Subspace Electrode Selection (right).
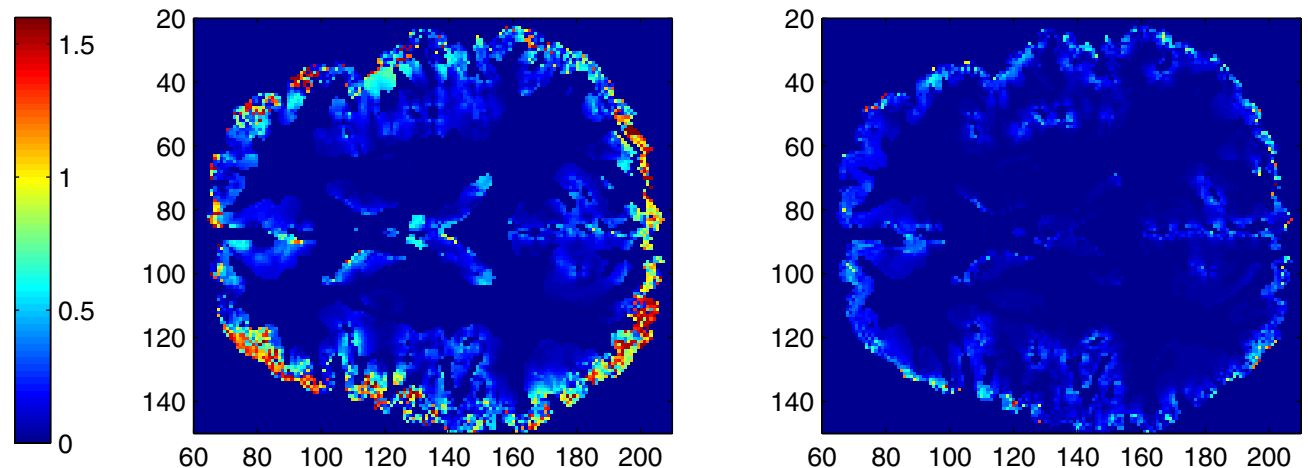

(b) Dipole orientation errors of dipoles oriented in the y-direction when using traditional method (left) and when using Subspace Electrode Selection (right).
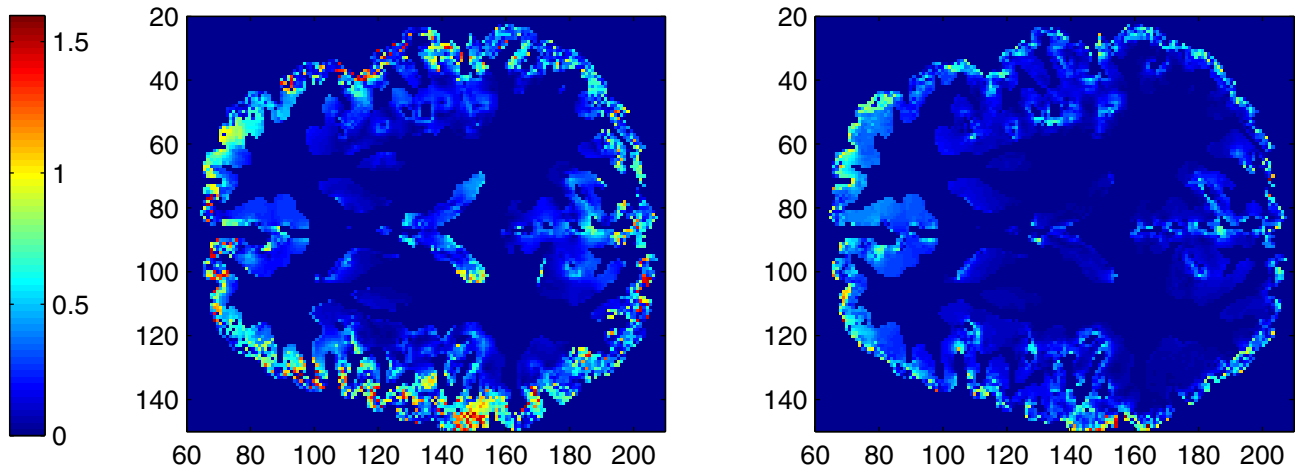

(c) Dipole orientation errors of dipoles oriented in the z-direction when using traditional method (left) and when using Subspace Electrode Selection (right).

Figure 11. Dipole orientation errors for (a) $x$-directed dipoles, (b) $y$-directed dipoles and (c) $z$-directed dipoles. 


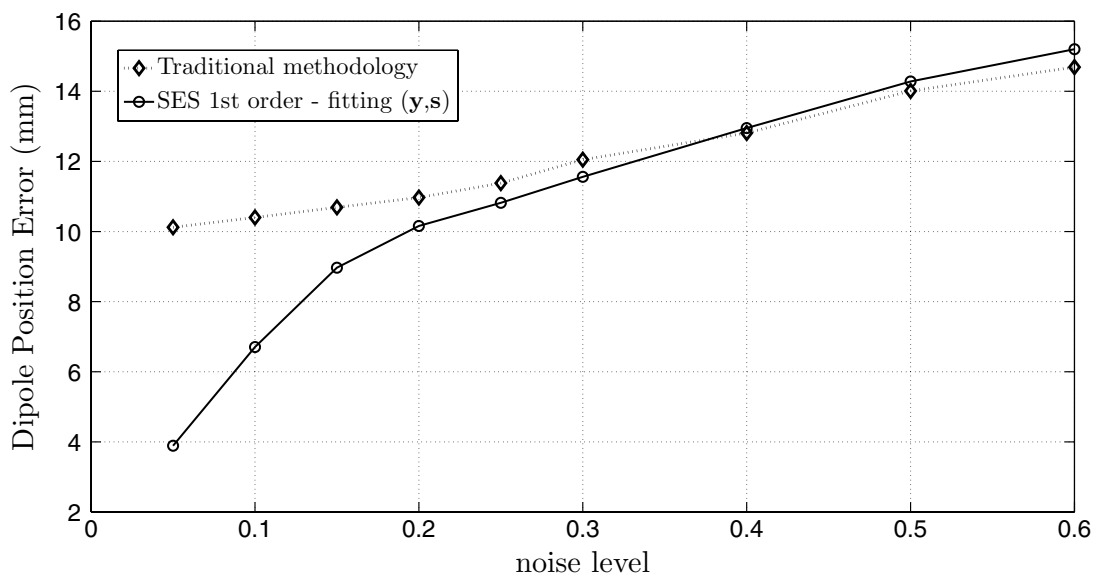

Figure 12. Dipole position error using the traditional methodology and SES methodology to the first and second order with incorporation of noise in simulation study. The fitting procedure in SES consists in fitting the data sets $\mathbf{y}$ to $\mathbf{s}$. The used data sets were generated for actual conductivity ratio $\xi_{\text {act }}=1 / 20$ and the inverse problem is solved for each data point 50 times using a forward model with assumed conductivity ratio $\widetilde{\xi}=1 / 60$.

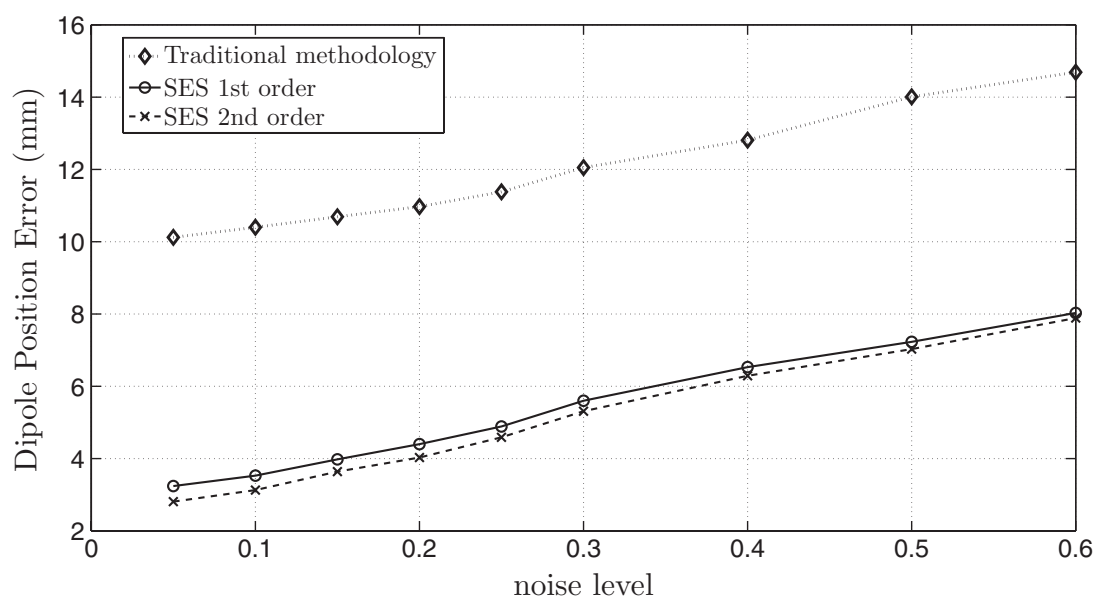

Figure 13. Dipole position error using the traditional methodology and SES methodology to the first and second order with incorporation of noise in simulation study. The fitting procedure in SES uses data sets $\mathbf{s}_{\text {sub }}$ and $\mathbf{u}$. The used data sets were generated for actual conductivity ratio $\xi_{\text {act }}=1 / 20$ and the inverse problem is solved for each data point 50 times using a forward model with assumed conductivity ratio $\widetilde{\xi}=1 / 60$.

procedure is used that is based on the principal vectors of the lead field and measured EEG potentials.

\subsection{Multiple-dipole localization}

We also investigated the errors made by a limited number of multiple dipoles. For given $p$ test dipoles, which are simultaneously active, with certain positions $\mathbf{r}_{d, \text { act, } k}$ and time-varying dipole 


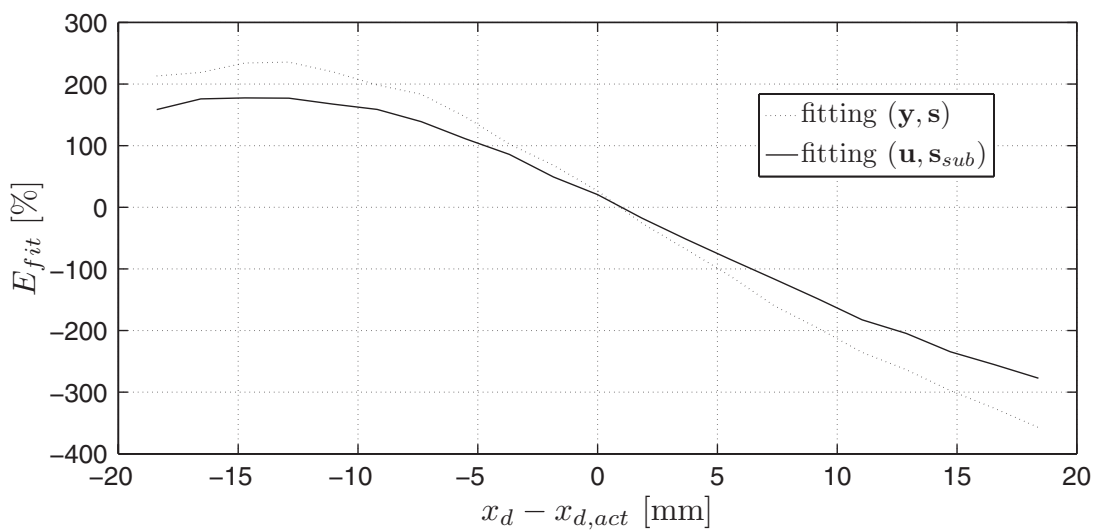

Figure 14. $E_{\text {fit }}$ of $\alpha$ as a function of $\mathbf{r}_{d}-\mathbf{r}_{d \text {,act }}$ along the $x$-axis for $y_{d} \equiv y_{d \text {,act }}$ and $z_{d} \equiv z_{d \text {,act }}$. $\xi_{\text {act }}-\widetilde{\xi}$ is 0.0259 in this simulation study and the constants were fitted for 50 different data samples.

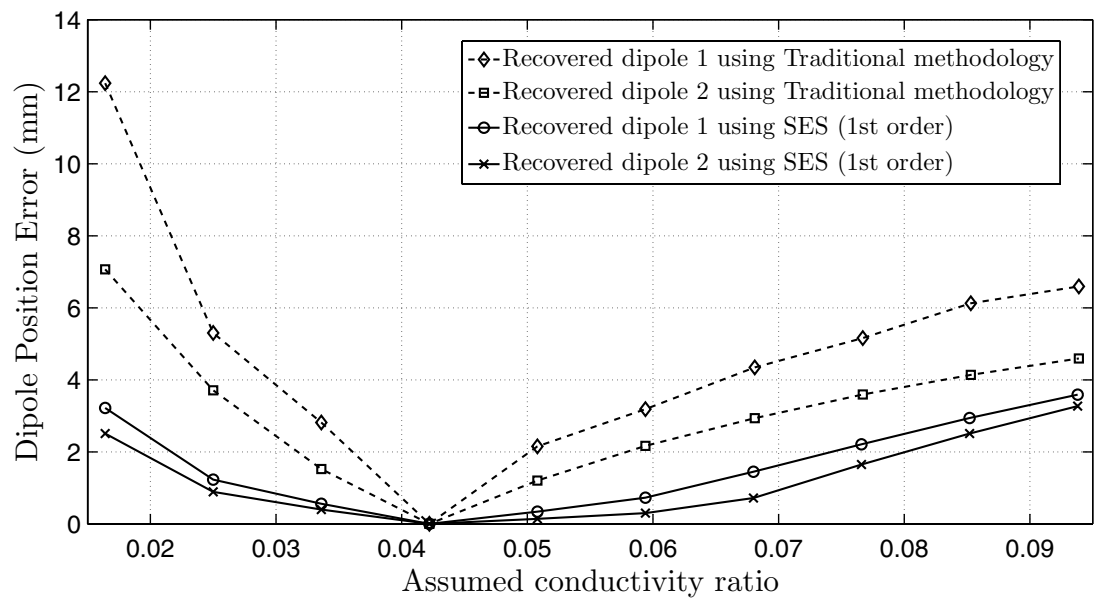

Figure 15. Dipole position errors for two dipoles using the traditional RAP-MUSIC algorithm and the first-order SES methodology.

orientations $\mathbf{D}_{\text {act, } k}(k=1, \ldots, p)$, the forward problem was solved for the actual conductivity ratio in the realistic head model, yielding a spatio-temporal EEG potential set. We assumed the time courses of the multiple dipoles as epileptic spikes or as sinusoidal time courses (with frequency 10,12 or $15 \mathrm{~Hz}$ ). $n=200$ time steps were considered, which corresponds with $1 \mathrm{~s}$ of EEG data. The inverse problem is then solved using a certain assumed conductivity ratio value. In this simulation study, inverse problems were solved for two dipoles (one spike and one sinusoidal varying dipole of frequency $10 \mathrm{~Hz}$ ), three dipoles (one spike and two sinusoidal with frequencies 10 and $12 \mathrm{~Hz}$ ), four dipoles (two spikes and two sinusoidal with frequencies 10 and $15 \mathrm{~Hz}$ ) and five dipoles (two spikes and three sinusoidal with frequencies 10, 12 and $15 \mathrm{~Hz}$ ) each time equally distributed in the head.

Figures 15 and 16 show the errors made when recovering two dipoles using the traditional, SES of first and second orders. We observe again a decrease in dipole position error and the advantage of using the second order versus the first order is relatively negligible. The results for 


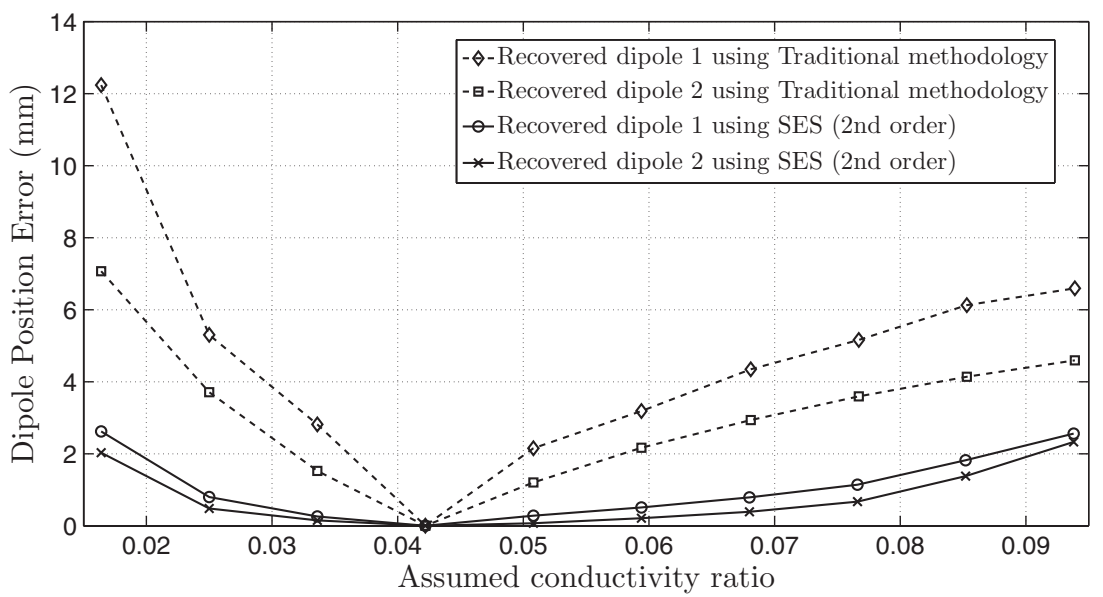

Figure 16. Dipole position errors for two dipoles using the traditional RAP-MUSIC algorithm and the second-order SES methodology.

Table 1. Dipole position error with the number of dipoles $p$ varying from 2 to 5 . The percentage decrease in dipole position error of the used first-order SES compared to the traditional methodology is depicted in the case of no noise and noise of level 0.2 in the data set.

\begin{tabular}{lll}
\hline$p$ & $\%$ decrease (no noise) & $\%$ decrease $(0.2$ noise level) \\
\hline 2 & 68.49 & 63.32 \\
3 & 64.28 & 59.63 \\
4 & 61.30 & 57.10 \\
5 & 62.51 & 55.98 \\
\hline
\end{tabular}

resolving more than two dipoles are shown in table 1 for clarity. We depict here the percentage decrease in the dipole position error, which is calculated as $100 \frac{E_{\text {Traa }}-E_{\text {EES }}}{E_{\text {Trad }}}$ with $E_{\text {Trad }}$ being the average dipole position errors of the $p$ used dipoles. $E_{\mathrm{SES}}$ is calculated in the same way. The added noise set is again white Gaussian noise.

From this table we can observe that the decrease in error is smaller for a larger number of dipoles but remains stable. The reason for this stability is because the RAP-MUSIC-based SES methodology uses in each maximization of the RAP-MUSIC cost function the whole set of electrodes where selection is then carried upon. The noise robustness of the methodology is shown in the third column where the percentage decrease stays stable. The inverse problem was solved starting from 20 noisy data sets with same noise level of 0.2.

This paper validates the methodology using a simulation study on a patient-specific head model. An experimental validation could be performed in the following way: application of the presented methodology onto measured EEG potentials in patients that were suffering from focal refractory epilepsy where the epileptic zone was surgically resected and the patient rendered seizure free. Comparison of the SES-based recovered zone with the resected zone can give an experimental validation of the methodology.

\subsection{Cost function surface and minimization path}

Due to the use of the SES cost functions (37), (38), the landscape is different than the traditional cost functions. Figures 17 and 18 show the cost function surface of the traditional 


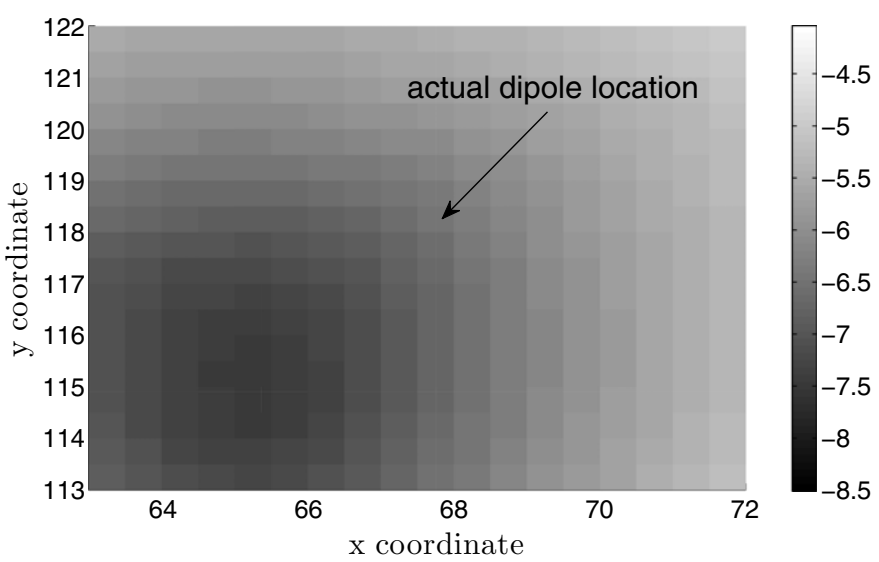

Figure 17. Traditional RRE lognormal cost function surface near actual dipole $\mathbf{r}_{d \text {, act }}=$ $[68,118,130]$ (arrow) with the EEG sample generated by actual $\xi_{\text {act }}=1 / 20$ and solved using assumed $\widetilde{\xi}=1 / 40$. This $2 \mathrm{D}$ slice is calculated at $z=130$.

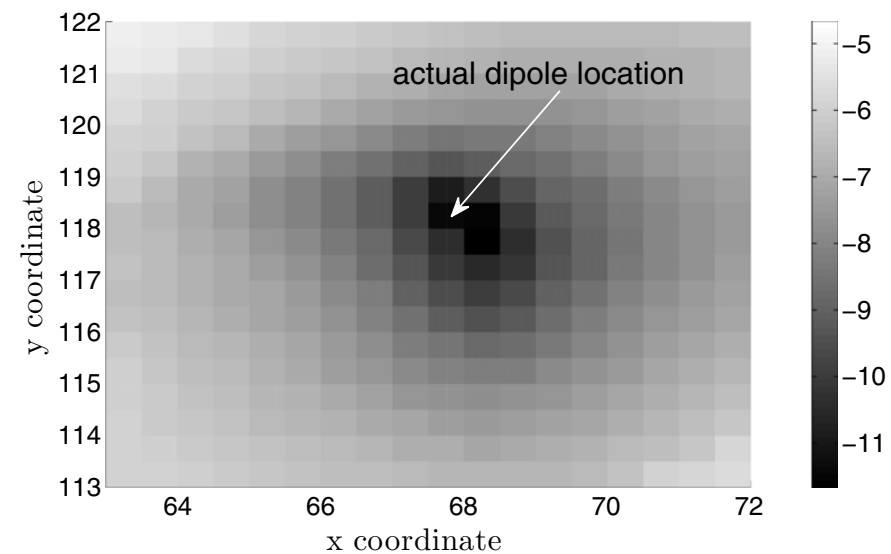

Figure 18. SES lognormal cost function surface near actual dipole $\mathbf{r}_{d \text {, act }}=[68,118,130]$ (arrow) with the EEG sample generated by actual $\xi_{\text {act }}=1 / 20$ and solved using assumed $\tilde{\xi}=1 / 40$. This $2 \mathrm{D}$ slice is calculated at $z=130$.

least-squares cost function and of the SES cost function surface, respectively. These measured EEG potentials $\mathbf{v}_{m}\left(\mathbf{r}_{d \text {, act }}, \mathbf{d}_{\text {act }}, \xi_{\text {act }}\right)$ were generated for dipole location $\mathbf{r}_{d \text {, act }}=[68,118,130]$, dipole orientation $\mathbf{d}_{\text {act }}=[0.5,0.4,0.7]$ with actual conductivity ratio $\xi_{\text {act }}=1 / 20$. The traditional cost function $\mathcal{Y}\left(\mathbf{r}_{d}, \widetilde{\xi}\right)$ and the first-order SES cost function $\mathcal{Y}^{\operatorname{SES}}\left(\mathbf{r}_{d}, \widetilde{\xi}\right)$ were calculated for every voxel in the neighborhood of $\mathbf{r}_{d \text {, act }}$ with assumed $\widetilde{\xi}=1 / 40$. When comparing these figures, we can observe that the landscape of the cost function is altered. Moreover, the minimum of the cost function is located in the SES case closer to the actual dipole location.

Moreover, figure 19 shows the path of the iterations $\mathbf{r}_{d}^{(l)}$ during the minimization of the traditional and SES cost functions. SES follows a certain minimum path of uncertainty, i.e. the iterations are least affected by the uncertainty. 


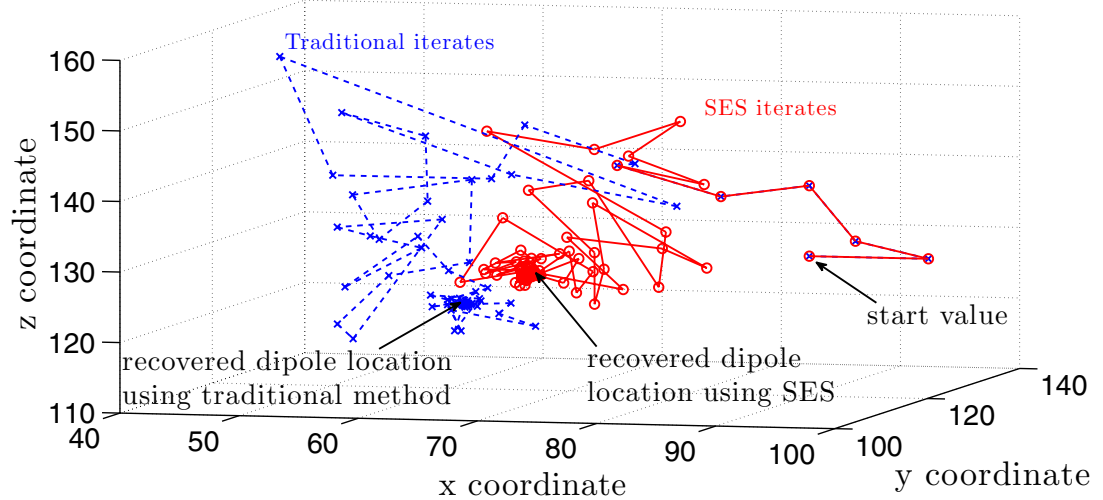

Figure 19. Path followed by the minimization procedure when minimizing the traditional and SES cost function.

\subsection{Drawbacks of SES}

The major drawback of the proposed methodology is that the minimization is highly sensitive upon the start value of the dipole. This is also the case when using the traditional minimization method since the traditional cost function is non-convex and may contain many local minima (Crevecoeur et al 2008). In the SES cost function this is also the case where additionally due to the internal fitting procedure, the cost function is significantly altered. Indeed, if the $\alpha$ parameter is changed to a large extent from one iteration to another, the changed SES cost function has difficulty to reach the global minimum. Further theoretical work is needed for stabilizing the minimization. A possible means is the use of trust region methodologies. A second drawback of the SES methodology is that it is much more time demanding compared to the traditional approach. The needed computational time for the minimization procedure is on average $28 \mathrm{~s}$ on a $2.4 \mathrm{GHz} \mathrm{PC}$, approximately $44 \%$ larger than the traditional method due to the internal fitting procedure and the calculation of the sensitivities in each iteration. For the second-order SES, the time is even more time demanding. Thirdly, the SES procedure needs more memory because the sensitivity calculations are based on the finite differentiation of lead fields that are calculated for different conductivity ratios in the neighborhood of the assumed conductivity ratio.

\section{Conclusion}

When solving the EEG inverse problem, a cost function needs to be minimized. For EEG dipole source reconstruction this is the widely used traditional least-squares cost function of the measured EEG potentials versus the calculated EEG potentials. Because the calculated EEG potentials are sensitive to the conductivity values, the uncertain conductivity values influence the recovered source estimates. In this paper, we introduced the subspace electrode selection (SES) methodology for the reduction of the effect of the uncertain conductivity values on the inverse solutions. SES uses the following basic operations: (a) redefinition of the cost function with inclusion of the sensitivity of the forward model to the uncertain conductivity ratio and (b) the selection of electrodes that are least affected by the conductivity ratio. The results show that the method enhances the source localization depending on the position of the dipoles, noise in measurements and the deviation of the assumed conductivity ratio value to 
the actual conductivity ratio. Special care is needed with respect to the internal fitting procedure within the redefined cost function, but fitting the principal vector to their sensitivities of the subspace correlation function of the measured EEG potentials and the lead field matrix.

Further research can be concentrated on decreasing the influence of multiple uncertainties on EEG dipole analysis and on incorporating other uncertainties such as electrode misplacements, and geometrical and source modeling errors. However, the uncertainty of the conductivity has the largest impact onto EEG source analysis. More research can be done when incorporating anisotropic conductivities in the head model with uncertain absolute conductivity values. The methodology has the potential to be applied onto other imaging modalities where the measurements need to be interpreted with a numerical model where uncertainties are included. The presented method can be valuable for brain research where as accurate as possible neural source specifications need to be recovered starting from EEG.

\section{Acknowledgments}

The authors wish to thank Hans Hallez and Bertrand Yitembe, and gratefully acknowledge the financial support of GOA07/GOA/006 and IUAP P6/21. GC is a postdoctoral researcher of the FWO.

\section{References}

Akhtari M, Mandelkern M, Bui D, Salamon N, Vinters H V and Mathern G W 2010 Variable anisotropic brain electrical conductivities in epileptogenic foci Brain Topogr. 23 292-300

Bai X and He B 2006 Estimation of number of independent brain electric sources from the scalp EEGs IEEE Trans. Biomed. Eng. 53 1883-92

Baillet S, Mosher J C and Leahy R M 2001 Electromagnetic brain mapping IEEE Signal Process. Mag. 18 14-30

Barrett R, Berry M, Chan T, Demmel J, Donato J, Dongarra Eijkhout V, Pozo R, Romine C and der Vorst H 1994 Templates for the Solution of Linear Systems (Philadelphia: SIAM)

Brodbeck V, Lascano A M, Spinelli L, Seeck M and Michel C M 2009 Accuracy of EEG source imaging of epileptic spikes in patients with large brain lesions Clin. Neurophysiol. 120 679-85

Chen F, Hallez H and Staelens S 2010 Influence of skull conductivity perturbations on EEG dipole source analysis Med. Phys. 37 4475-84

Crevecoeur G, Hallez H, Van Hese P, D’Asseler Y, Dupré L and Van de Walle R 2008 A hybrid algorithm for solving the EEG inverse problem from spatio-temporal EEG data Med. Biol. Eng. Comput. 46 767-77

de Munck J C, Van Dijk B W and Spekreijse H 1988 Mathematical dipoles are adequate to describe realistic generators of human brain activity IEEE Trans. Biomed. Eng. 35 960-5

Friston K J (ed) 2007 Statistical Parametric Mapping: The Analysis of Functional Brain Images (New York: Academic)

Gabriel C 2005 Dielectric properties of biological tissue: variation with age Bioelectromagnetics 26 S12-8

Gaignaire R, Crevecoeur G, Dupré L, Sabariego R V, Dular P and Geuzaine C 2010 Stochastic uncertainty quantification of the conductivity in EEG source analysis by using polynomial chaos decomposition IEEE Trans. Magn. 46 3457-60

Geddes L A and Baker LE 1967 The specific resistance of biological material: a compendium of data for the biomedical engineer and physiologist Med. Biol. Eng. 5 271-93

Gençer N G and Acar C E 2004 Sensitivity of EEG and MEG measurements to tissue conductivity Phys. Med. Biol. 49 701-17

Golub G H and Loan C F V 1996 Matrix Computations 3rd edn (Baltimore, MD: John Hopkins University Press) p 604

Gonçalves S I, de Munck J C, Verbunt J P A, Bijma F, Heethaar R M and Lopes da Silva F 2003 In vivo measurement of the brain and skull resistivities using an EIT-based method and realistic models for the head IEEE Trans. Biomed. Eng. 50 754-67

Hallez H, Vanrumste B, Van Hese P, D’Asseler Y, Lemahieu I and Van de Walle R 2005 A finite difference method with reciprocity used to incorporate anisotropy in electroencephalogram dipole source localization Phys. Med. Biol. 50 3787-806 
Hallez H, Vanrumste B, Van Hese P, Delputte S and Lemahieu I 2008 Dipole estimation errors due to differences in modeling anisotropic conductivities in realistic head models for EEG source analysis Phys. Med. Biol. 53 1877-94

Haueisen J, Ramon C, Eiselt M, Brauer H and Nowak H 1997 Influence of tissue resistivities on neuromagnetic fields and electric potentials studied with a finite element model of the head IEEE Trans. Biomed. Eng. 44 727-35

Haueisen J, Tuch D S, Ramon C, Schimpf P H, Wedeen V J, George J S and Belliveau J W 2002 The influence of brain tissue anisotropy on human EEG and MEG NeuroImage 15 159-66

Hoekema R, Wieneke G H, Leijten F S, van Veelen C W M, van Rijen P C, Huiskamp G J, Ansems J and van Huffelen A C 2003 Measurement of the conductivity of skull, temporarily removed during epilepsy surgery Brain Topogr. 16 29-38

Huang M, Aine C, Supek S, Best E, Ranken D and Flynn E 1998 Multi- start downhill simplex method for spatiotemporal source localization in magnetoencephalography Electroencephalogr. Clin. Neurophysiol. $10832-44$

Knösche T, Berends E, Jagers H and Peters M 1998 Determining the number of independent sources of the EEG: a simulation study on information criteria Brain Topogr. 11 111-24

Laarne P, Kauppinen P, Hyttinen J, Malmivuo J and Eskola H 1999 Effects of tissue resistivities on electroencephalogram sensitivity distribution Med. Biol. Eng. Comput. 37 555-9

Laarne P H, Tenhunen-Eskelinen M L, Hyttinen J K and Eskola H J 2000 Effect of EEG electrode density on dipole localization accuracy using two realistically shaped skull resistivity models Brain Topogr. 12 249-54

Lai Y, van Drongelen W, Ding L, Hecox K E, Towle V L, Frim D M and He B 2005 Estimation of in vivo human brain-to-skull conductivity ratio from simultaneous extra- and intra-cranial electrical potential recordings Clin. Neurophysiol. 116 456-65

Lew S, Wolters C H, Anwander A, Makeig S and MacLeod R S 2007 Low resolution conductivity estimation to improve source localization New Frontiers in Biomagnetism Proc. 15th Int. Conf. Biomagnetism pp 149-52

Liu H and Schimpf P H 2006 Efficient localization of synchronous EEG source activities using a modified RAPMUSIC algorithm IEEE Trans. Biomed. Eng. 53 652-61

Mosher J C and Leahy R M 1999 Source localization using recursively applied and projected (RAP) MUSIC IEEE Trans. Signal Process. 37 332-40

Oostendorp T F, Delbeke J and Stegeman D F 2000 The conductivity of the human skull: results of in vivo and in vitro measurements IEEE Trans. Biomed. Eng. 47 1487-92

Pascual-Marqui R D 1999 Review of methods for solving the EEG inverse problem Int. J. Bioelectromagn. $175-86$

Plis S M, George J S, Jun S C, Ranken D M, Volegov P L and Schmidt D M 2007 Probabilistic forward model for electroencephalography source analysis Phys. Med. Biol. 52 5309-27

Pohlmeier R, Buchner H, Knoll G, Rienacker A, Beckmann R and Pesch J 1997 The influence of skullconductivity misspecification on inverse source localization in realistically shaped finite element models Brain Topogr. 9 157-62

Rush S and Driscoll D 1969 EEG electrode sensitivity-an application of reciprocity IEEE Trans. Biomed. Eng. 16 15-22

Sijbers J, Vanrumste B, Van Hoey G, Boon P and Verhoye M 2000 Automatic localization of EEG electrode markers within 3D MR data Magn. Reson. Imag. $18485-8$

Steinsträter O, Sillekens S, Junghoefer M, Burger M and Wolters C H 2010 Sensitivity of beamformer source analysis to deficiencies in forward modeling Hum. Brain Mapp. 31 1907-27

Vallaghé S and Clerc M 2009 A global sensitivity analysis of three- and four-layer EEG conductivity models IEEE Trans. Biomed. Eng. 56 988-95

Vallaghé S, Clerc M and Badier J-M 2007 In vivo conductivity estimation using somatosensory evoked potentials and cortical constraint on the source Proc. 4th IEEE Int. Symp. Biomedical Imaging pp 1036-9

Vanrumste B, Van Hoey G, Van de Walle R, D'Havé M, Lemahieu I and Boon P 2000 Dipole location errors in electroencephalogram source analysis due to volume-conductor model errors Med. Biol. Eng. Comput. 38 528-34

Vanrumste B, Van Hoey G, Van de Walle R, D’Havé M, Lemahieu I and Boon P 2001 The validation of the finite difference method and reciprocity for solving the inverse problem in EEG dipole source analysis Brain Topogr. 14 83-92

Vanrumste B, Van Hoey G, Van de Walle R, D’Havé M, Lemahieu I and Boon P 2002 Comparison of performance of spherical and realistic head models in dipole localization from noisy EEG Med. Eng. Phys. 24 403-18

Wolters C H, Anwander A, Tricoche X, Weinstein D, Koch M A and MacLeod R S 2006 Influence of tissue conductivity anisotropy on EEG/MEG field and return current computation in a realistic head model: a simulation and visualization study using high-resolution finite element modeling NeuroImage 30 813-26 